Noble, George $\mathrm{H}$.

Ferns, Fern Allies and Flowering Plants of Rhode Island

QK

523.3 


\section{DUKE UNIVERSITY}

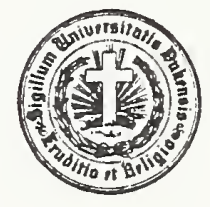

LIBRARY 

Digitized by the Internet Archive in 2016 with funding from Duke University Libraries 



\title{
THE FERNS, FERN ALLIES AND FLOWERING PLANTS OF RHODE ISLAND
}

A Revision of the first fifty-eight pages of

James L. Bennett's "Plants of Rhode Island"

published by the

Providence Franklin Society in 1888

\author{
PUBLISHED BY THE \\ PROVIDENCE FRANKLIN SOCIETY
}

1920 


\section{INTRODUCTION}

$F^{O R}$ many years a revision of Bennett's "Plants of Rhode $F$ Island" has been desired. Many plants should be added to his list and the modern system of nomenclature and classification (Gray's New Manual of Botany, Seventh Edition) requires a complete rearrangement.

The Standing Committee of the Providence Franklin Society was instructed by the society after a most interesting lecture by Miss Eda M. Round to prepare the revision. This revision is the work of the late George H. Noble, a member of the Committee. He prepared the manuscript and verified the items. He completed this task a few days before his death.

It has been evident from his notes and correspondence that especial thanks are due to Miss Eda M. Round of the Providence Technical High School, Miss Marie E. Gaudette of the Roger WilliamsPark Museum, and Professor J. Franklin Collins of Brown University. The Committee is indebted to David W. Hoyt, Principal Emeritus of the Providence English High School for assistance in examining the manuscript and letters left by Mr. Noble and to Dr. Henry P. Lovewell for assistance in reading the proof.

Standing Committee of the

Prooidence Franklin Society

ERNEST R. HAGER

DANIEL L. MATHEWSON WILLIAM W. ESTES 


\title{
DIVISION 1. PTERIDOPHYTA
}

\author{
(Ferns and Fern Allies)
}

ORDER I FILICALES

FAMILY 2. POLYPODIACEAE (Fern Family)

1. POL YPODIUM (Tourn.) L. Polypody

1. vulgare L. ${ }^{1}$

vulgare var. cambricum (L.) Willd. ${ }^{1}$

2. PHEGOPTERIS (Presl.) Fée. Beech Fern

1. polypodioides Fée. ${ }^{1}$

2. hexagonoptera (Michx.) Fée. ${ }^{1}$

3. Dryopteris (L.) Fée. ${ }^{1}$ (Oak Fern.)

4. ADIANTUM (Tourn.) L. Maidenhair

1. pedatum $L .{ }^{1}$

5. PTERIS L. Brake or Bracken

1. aquilina L. ${ }^{1}$ (Common Brake.)

7. PELLAEA Link. Cliff Brake

1. atropurpurea (L.) Link. ${ }^{3}$

9. WOODWARDIA Sm. Chain Fern

1. virginica (L.) $\mathrm{Sm} .^{1}$

2. areolata (L.) Moore. ${ }^{52}$ W. angustifolia Sm. ${ }^{1}$

10. ASPLENIUM L. Spleenwort

3. Trichomanes L. ${ }^{1}$

5. platyneuron (L.) Oakes. A. ebeneum Ait. ${ }^{1}$

10. acrostichoides Sw. A. thelepteroides Michx. ${ }^{1}$

11. Filix-femina (L.) Bernh. ${ }^{1}$ (Lady Fern.)

12. CAMPTOSORUS Link. Walking Leaf

1. rhizophyllus (L.) Link. ${ }^{1}$

13. POLYSTICHUM Roth.

1. acrostichoides (Michx.) Schott. (Christmas Fern.) Aspidium Sw. ${ }^{1}$

acrostichoides var. Schweinitzii (Beck.) Small. Aspidium acrostichoides var. incisum Gray. ${ }^{1}$ 
14. ASPIDIUM Sw. Shield Fern. Wood Fern

1. Thelypteris (L.) $\mathrm{Sw}^{34}$

2. simulatum Davenp. ${ }^{32-34}$

3. noveboracense (L.) $\mathrm{Sw}_{{ }^{3}}{ }^{3}$

5. marginale (L..) Sw. ${ }^{1}$

8. Boottii Tuckerm. A. spinulosum var. Boottii. ${ }^{1}$

9. cristatum (L.) Sw. $^{1}$ cristatum var. Clintonianum D. C. Eaton. ${ }^{1}$

10. spinulosum (O. F. Müller) $\mathrm{Sw}{ }^{34}$ spinulosum var. intermedium (Muhl.) D. C. Eaton. ${ }^{1}$

15. CYSTOPTERIS Bernh. Bladder Fern

1. bulbifera (L.) Bernh. ${ }^{1}$

2. fragilis (L.) Bernh. ${ }^{1}$

16. WOODSIA R. Br.

1. ilvensis (L.) $\mathrm{R} . \mathrm{Br}^{1}{ }^{1}$

4. obtusa (Spreng.) Torr. ${ }^{1}$

17. DICKSONIA L'Hér.

1. punctilobula (Michx.) Gray. ${ }^{1}$ (Hay-scented Fern.)

18. ONOGLEA L.

1. sensibilis L. ${ }^{1}$ (Sensitive Fern.) sensibilis var. obtusilobata (Schkuhr.) Torr. ${ }^{1}$

2. struthiopteris (L.) Hoffm. O. germanica Hook. ${ }^{1}$

FAMILY 3. SGHIZAEACEAE (Curly Grass Family)

2. LYGODIUM Sw. Climbing Fern

1. palmatum (Bernh.) Sw. ${ }^{1}$

FAMILY 4. OSMUNDACEAE (Flowering Fern Family)

1. OSMUNDA (Tourn.) L. Flowering Fern

1. regalis $\mathrm{L}^{1}$ (Flowering Fern.)

2. Claytoniana L. ${ }^{1}$ (Interrupted Fern.)

3. cinnamomea L. ${ }^{1}$ (Cinnamon Fern.) cinnamomea var. glandulosa Waters. ${ }^{32}$ cinnamomea var. frondosa Gray. ${ }^{1}$

FAMILY 5. OPHIOGLOSSACEAE (Adder's Tongue Family)

1. OPHIOGLOSSUM (Tourn.) L. Adder"s Tongue 1. vulgatum $\mathrm{L}$. $O$. vulgare $\mathrm{L}$. $^{1}$ 
2. BOTR YCHIUM Sw. Moonwort

5. obliquum Muhl. B. ternatum var. Milde. ${ }^{1}$ obliquum var. dissectum (Spreng.) Clute. B. ternatum var. dissectum Milde. ${ }^{1}$

6. ternatum (Thunb.) Sw. var. intermedium D. C. Eaton. ${ }^{3}$ ternatum var. rutaefolium (A. Br.) D. C. Eaton. ${ }^{3}$

7. virginianum (L.) Sw. (Rattlesnake Fern.) B. Virginicum L. ${ }^{1}$

\section{ORDER II EQUISETALES}

FAMILY 8. EQUISETACEAE (Horsetail Family)

1. EQUISETUM (Tourn.) L. Horsetail

1. arvense L. ${ }^{1}$ (Common H.)

3. sylvaticum L. ${ }^{1}$

6. fluviatile L. (Pipes.) E. limosum L. ${ }^{1}$

8. hyemale L. ${ }^{1}$ (Scouring Rush.)

\section{ORDER III LYCOPODIALES}

FAMILY 9. LYCOPODIACEAE (Club Moss Family)

\section{LYCOPODIUM L. Club Moss}

2. Iucidulum Michx. ${ }^{34}$

4. inundatum $L^{3 .-52}$

5. annotinum $L .{ }^{1}$

6. clavatum L. ${ }^{1}$ (Common C.)

7. obscurum L. ${ }^{34}$

obscurum var. dendroideum (Michx.) D. C. Eaton. L. dendroideum Michx. ${ }^{1}$

11. complanatum L. ${ }^{34}$

FAMILY 10. SELAGINELLAGEAE

1. SELAGINELLA Beauv.

2. rupestris (L.) Spring. ${ }^{1}$

3. apus (L.) Spring. ${ }^{1}$

FAMILY 11. ISOËTACEAE (Quillwort Family)

1. ISOETES L. Quillwort

2. Tuckermani A. Br. ${ }^{1}$

5. riparia Engelm. ${ }^{1}$

7. echinospora Dur. ${ }^{1}$

11. Engelmanni A. Br. ${ }^{1}$

Engelmanni var. gracilis Engelm. ${ }^{1}$ 


\section{DIVISION II. SPERMATOPHYTA}

(Seed Plants, Phanerogamia, or Flowering Plants)

Subdivision I Gymnospermae

ORDER IV CONIFERALES

FAMILY 12. TAXACEAE (Yew Family)

1. TAXUS (Tourn.) L. Yew

1. canadensis Marsh. (American Y., Ground Hemlock.) T. baccata L. var. canadensis. ${ }^{1}$

FAMILY 13. PINACEAE (Pine Family)

1. PINUS (Tourn.) L. Pine

1. Strobus L. ${ }^{1}$ (White P.)

3. rigida Mill. ${ }^{1}$ (Pitch $\mathrm{P}$. )

10. resinosa Ait. ${ }^{1}$ (Red P.)

2. LARIX (Tourn.) Adans. Larch

1. laricina (Du Roi) Koch. (American or Black L., Tamarack, Hackmatack.) L. americana Michx. ${ }^{1}$

3. PICEA Link. Spruce

1. canadensis (Mill.) B S P. (White or Cat S.)

3. mariana (Mill.) B S P. ${ }^{23}$ (Black or Bog S.) P. nigra Lk. ${ }^{1}$

4. ABIES (Tourn.) Hill. Fir

1. balsamea (L.) Mill. (Balsam or Balm-of-Gilead F.)

5. TSUGA (Endl.) Carr. Hemlock

1. canadensis (L.) Carr. ${ }^{1}$

7. CHAMAECYPARIS Spach. White Cedar. Cypress.

1. thyoides (I.) B S P. (White Cedar.) C. sphaeroidea Spach. ${ }^{1}$

8. THUJA L. Arbor Vitae

1. occidentalis L. (Arbor Vitae, White Cedar.)

9. JUNIPERUS (Tourn.) L. Juniper

1. communis L. ${ }^{1}$ (Common J.) 
2. horizontalis Moench. J. sabina var. procumbens Pursh. ${ }^{1}$

3. virginiana L. ${ }^{1}$ (Red Cedar or Savin.)

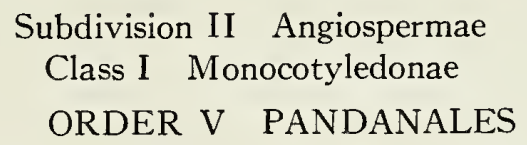

FAMILY 14. TYPHACEAE (Cat-tail Family)

1. TYPHA (Tourn.) L. Cat-tail Flag

1. latifolia L. ${ }^{1}$ (Common Cat-tail.)

2. angustifolia L. ${ }^{1}$

FAMILY 15. SPARGANIACEAE (Bur-reed Family)

1. SPARGANIUM (Tourn.) L. Bur-reed

1. eurycarpum Engelm.$^{33}$

2. americanum Nutt. . $^{33-34}$

americanum var. androcladum (Engelm.) Fernald \& Eames. ${ }^{3-31-37}$

6. simplex Huds. ${ }^{1}$

ORDER VI NAJADALES

FAMILY 16. NAJADACEAE (Pondweed Family)

1. POTAMOGETON (Tourn.) L. Pondweed

1. natans $L^{39}$

2. Oakesianus Robbins. ${ }^{39}$

4. epihydrus Raf. ${ }^{39}$ P. Claytonii Tuckerm. ${ }^{1}$

6. americanus C. \&. S. ${ }^{39} \quad P$. lonchites Tuckerm. ${ }^{1}$

7. pulcher Tuckerm. ${ }^{39}$

8. amplifolius Tuckerm. ${ }^{39}$

10. heterophyllus Schreb. ${ }^{39}$ Gramineus L. var. ${ }^{1}$

heterophyllus forma Graminifolius (Fries.) Morong. ${ }^{39}$

Gramineus L. var. Graminifolius. ${ }^{1}$

heterophyllus forma longipedunculatus (Merat.) Morong. heterophyllus forma myriophyllus (Robbins) Morong. ${ }^{39}$

Gramineus L. var. myriophyllus. ${ }^{1}$

15. perfoliatus L. ${ }^{1}$

16. bupleuroides Fernald. ${ }^{39}$

19. zosterifolius Schumacher. ${ }^{39} \quad P$. compressus L. ${ }^{1}$

25. pusillus L. ${ }^{39}$

27. Vaseyi Robbins. ${ }^{1}$ 
28. gemmiparus Robbins. ${ }^{39}$

30. foliosus Raf. ${ }^{39}$ P. pauciflorus Pursh. ${ }^{1}$

31. confervoides Reichenb. P. Tuckermani Robbins. ${ }^{1}$

32. hybridus Michx. ${ }^{39}$

33. dimorphus Raf. ${ }^{39}$ P. spirillus Tuck. ${ }^{1}$

36. pectinatus L. ${ }^{39}$

38. Robbinsii Oakes. ${ }^{39}$

\section{RUPPIA L. Ditch Grass}

1. maritima L. ${ }^{39}$

3. ZANNICHELLIA (Mich.) L. Horned Pondweed 1. palustris $\mathrm{L} .{ }^{39}$

4. ZOSTERA L. Grass Wrack. Eel Grass

1. marina L. ${ }^{39}$

5. NAJAS L. Naiad

2. flexilis (Willd.) Rostk. \& Schmidt. ${ }^{39}$

4. gracillima (A. Br.) Magnus. ${ }^{39}$

FAMILY 17. JUNCAGINACEAE (Arrow Grass Family) 1. SCHEUCHZERIA L.

1. palustris $\mathrm{L} .{ }^{39}$

\section{TRIGLOCHIN L. Arrow Grass}

2. maritima L. ${ }^{37-39}$

3. palustris $\mathrm{L}^{1}$

FAMILY 18. ALISMACEAE (Water-plantain Family)

1. SAGITTARIA L. Arrow-head

2. latifolia Willd. ${ }^{34}$ S. variabilis var. Engelm. ${ }^{1}$ latifolia forma obtusa (Muhl.) Robinson. S. variabilis Engelm. var. obtusa. ${ }^{1}$

latifolia forma hastata (Pursh.) Robinson. S. variabilis Engelm. var. hastata. ${ }^{1}$

latifolia forma diversifolia (Engelm.) Robinson. S. variabilis Engelm. var. diversifolia. ${ }^{1}$

3. Engelmanniana J. G. Sm. ${ }^{3-34}$ S. variabilis var. gracilis Engelm. ${ }^{1}$

8. heterophylla Pursh. ${ }^{1}$

heterophylla var. angustifolia Engelm. S. iariabilis Engelm. var. angustifolia. ${ }^{1}$

9. graminea Michx. ${ }^{1}$ 
2. LOPHOTOGARPUS Th. Durand.

2. calycinus (Engelm.) J. G. Sm. Sagittaria Engelm. ${ }^{1}$

4. ALISMA L. Water Plantain

1. Plantago-aquatica L. ${ }^{3}$ A. Plantago L. var. americanum. ${ }^{1}$

FAMILY 19. HYDROCHARITACEAE (Frog's Bit Family)

1. ELODEA Michx. Water-weed

1. canadensis Michx. Anacharis Planch. ${ }^{1}$

2. VALLISNERIA (Mich.) L. Tape Grass. Eel Grass

1. Spiralis L. ${ }^{1}$

ORDER VII GRAMINALES

FAMILY 20. GRAMINEAE (Grass Family)

1. TRIPSACUM L. Gama Grass. Sesame Grass

1. dactyloides L. ${ }^{1}$ T. dactyloides var. monostachyum Wood. ${ }^{1}$

4. ANDROPOGON (Royen.) L. Beard Grass

1. scoparius Michx. ${ }^{1}$

3. virginicus L. A. dissitiflorus Michx. ${ }^{1}$

6. furcatus Muhl. A. provincialis Lam. ${ }^{1}$

5. SORGHASTRUM Nash.

1. nutans (L.) Nash. (Indian Grass, Wood Grass.) $)^{1}$ Chrysopogon Benth. ${ }^{1}$

6. DIGITARIA Scop. Finger Grass

1. filiformis (L.) Koehler. Panicum L. ${ }^{1}$

*3. humifusa Pers. Panicum glabrum Gaud. ${ }^{1}$

*5. sanguinales (L.) Scop. (Crab Grass.) Panicum L. ${ }^{52}$

9. PASPALUM L.

4. setaceum Michx. ${ }^{52}$

11. laeve Michx. ${ }^{1}$

11. PANICUM L. Panic Grass

2. verrucosum Muhl. ${ }^{1}$

3. capillare L. ${ }^{52}$ (Old-witch Grass.)

6. philadelphicum Bernh. P. minus Nash. ${ }^{28}$

*7. miliaceum L. ${ }^{1}$ (European Millett.)

8. dichotomiflorum Michx. P. proliferum Lam. ${ }^{52}$

9. amarum Ell. ${ }^{1}$ 
11. virgatum L. ${ }^{52}$ (Switch Grass.)

13. agrostoides Spreng. ${ }^{1}$

17. depauperatum Muhl. ${ }^{1}$

21. xalapense H B K. P. laxiflorum Lam. ${ }^{1}$

27. dichotomum L. ${ }^{41} P$. ramulosum $\mathrm{Mx}^{1}$

28. barbulatum Michx. ${ }^{3}$

31. microcarpon Muhl. ${ }^{1}$

35. spretum Schultes. P. nitidum Lam. ${ }^{3}$

36. Lindheimeri Nash. ${ }^{41}$

39. implicatum Scribn. ${ }^{3}$

41. oricola Hitchic. \& Chase. ${ }^{48}$

53. columbianum Scribn. ${ }^{3}$

62. oligosanthes Schultes. P. pauciflorum Ell. ${ }^{1}$

71. clandestinum L. ${ }^{1}$

73. latifolium L. ${ }^{1}$

13. EGHINOGLOA Beauv.

*1. crusgalli (L.) Beauv. (Barnyard Grass.) Panicum L. ${ }^{1}$

2. Walteri (Pursh.) Nash. Panicum crusgalli var. Kispidum. ${ }^{1}$

14. SETARIA Beauv. Bristly Foxtail Grass

*2. glauca (L.) Beauv. ${ }^{1}$ (Foxtail, Pigeon Grass.)

*3. verticillata (L.) Beuav. ${ }^{1}$

*4. viridis (L.) Beauv. ${ }^{1}$ (Green F., Bottle Grass.)

*5. italica (L.) Beauv. ${ }^{1}$

15. GENCHRUS L. Sandbur. Bur Grass

2. tribuloides L. ${ }^{1}$

16. ZIZANIA (Gronov.) L. Water or Indian Rice 2. aquatica L. ${ }^{1}$

18. LEERSIA Sw. Cut-grass. White Grass

1. virginica Willd. ${ }^{1}$ (White Grass.)

2. oryzoides (L.) Sw. ${ }^{1}$ (Rice Cut-grass.)

19. PHALARIS L. Canary Grass

*1. canariensis L. ${ }^{1}$ (Canary Grass.)

2. arundinacea L. ${ }^{1}$ (Reed C.)

20. ANTHOXANTHUM L. Sweet Vernal Grass. *1. odoratum L. ${ }^{1}$ 
21. HIEROCHLOE (Gmel.) R. Br. Holy Grass

1. odorata (L.) Wahlenb. (Vanilla or Seneca Grass.) $H$. borealis R. \& S. ${ }^{1}$

22. MILIUM (Tourn.) L. Millet Grass

1. effusum L. ${ }^{1}$

23. ORYZOPSIS Michx. Mountain Rice

1. pungens (Torr.) Hitchc. O canadensis Torr. ${ }^{1}$

2. asperifolia Michx. ${ }^{1}$

3. racemosa $(\mathrm{Sm}$.$) Ricker. O. melanocarpa Muhl. { }^{1}$

24. STIPA L. Feather Grass

3. avenacea L..' (Black Oat Grass.)

4. comata Trin. \& Rupr. ${ }^{3}$

25. ARISTIDA L. Triple-awned Grass

1. dichotoma Michx. ${ }^{1}$ (Poverty Grass.)

4. gracilis Ell. ${ }^{1}$

9. purpurascens Poir. ${ }^{1}$

26. MUHLENBERGIA Schreb.

1. sobolifera (Muhl.) Trin. ${ }^{1}$

2. tenuiflora (Willd.) B S P. M. Willdenovii Trin. ${ }^{1}$

3. sylvatica Torr. ${ }^{1}$

5. mexicana (L.) Trin. ${ }^{2}$

6. racemosa (Michx.) B S P. M. glomerata Trin. ${ }^{1}$

7. Schreberi J. F. Gmel. (Drop-seed, Nimble Will.) $M$. diffusa Schreb. ${ }^{1}$

8. capillaris (Lam.) Trin. ${ }^{1}$ (Hair Grass.)

27. BRACHYELYTRUM Beauv.

1. erectum (Schreb.) Beauv. B. aristatum Beauv ${ }^{1}$

29. PHLEUM L.

*1. pratense L. ${ }^{1}$ (Timothy, Herd's Grass.)

30. ALOPECURUS L. Foxtail Grass

*1. pratensis L. ${ }^{1}$ (Meadow F.)

2. geniculatus L. ${ }^{1}$ (Floating F.)

geniculatus var. aristulatus Torr. ${ }^{1}$

31. SPOROBOLUS R. Br. Drop-seed. Rush Grass

3. asper (Michx.) Kunth. ${ }^{1}$ S. longifolius Wood. ${ }^{3}$

6. vaginiflorus (Torr.) Wood. ${ }^{1}$ 
10. cryptandrus (Torr.) Gray. B. V.

14. uniflorus (Muhl.) Scribn. \& Merr. S. serotina Gray. ${ }^{1}$

\section{AGROSTIS L. Bent Grass}

1. alba L. (Fiorin or White B., Red Top.) A. vulgaris var. With. ${ }^{1}$

alba var. vulgaris (With.) Thurb. (Red Top.) A. vulgaris With. ${ }^{1}$

3. hyemalis (Walt.) B S P. (Hair Grass.) A. scabra Willd. ${ }^{1}$

4. perennans (Walt.) Tuckerm. ${ }^{1}$ (Thin Grass.)

*5. canina L. ${ }^{1}$ (Brown Bent Grass.)

35. CALAMAGROSTIS Adans. Reed Bent Grass

4. canadensis (Michx.) Beauv. (Blue-joint Grass.) Deyeuxia Kunth. ${ }^{1}$

9. cinnoides (Muhl.) Barton. ${ }^{34}$ Deyeuxia Nuttaliana Steud. ${ }^{1}$

36. AMMOPHILA Host.

1. $\operatorname{arenaria~(L.)~Link.~}{ }^{1}$ (Sea Sand-reed, Marram, Psamma Beach Grass.)

38. CINNA L. Wood Reed Grass

1. arundinacea L. ${ }^{1}$

40. HOLCUS L.

*1. lanatus L. ${ }^{1}$ (Velvet Grass.)

41. SPHENOPHOLIS Scribn.

1. obtusata (Michx.) Scribn. Eatonia Raf. ${ }^{1}$

3. pallens (Spreng.) Scribn. Eatonia pennsylvanica Gray. ${ }^{1}$

4. palustris (Michx.) Scribn. Trisetum palustre L. ${ }^{1}$

44. DESCHAMPSIA Beauv. (Common Hair Grass.)

1. flexuosa (L.) Trin. ${ }^{1}$

45. AVENA (Tourn.) L. Oat

*4. sativa $\mathrm{L}^{3}{ }^{3}$

46. ARRHENATHERUM Beauv. Oat Grass

*1. elatius (L.) Beauv. (Tall O.) A. avenaceum Beauv. ${ }^{1}$

47. DANTHONIA D C. Wild Oat Grass

1. spicata (L.) Beauv. ${ }^{1}$

4. sericea Nutt. ${ }^{1}$ 
48. SPARTINA Schreb. Cord or Marsh Grass

1. Michauxiana Hitchc. (Slough Grass.) S. cynosuroides Willd. ${ }^{1}$

2. cynosuroides (L.) Roth. (Salt Reed Grass.) S. polystachyon Willd. ${ }^{1}$

3. glabra Muhl. var. pilosa Merr. S. stricta Roth, ${ }^{1}$

4. patens var. juncea (Michx.) Hitchc. S. juncea Willd. ${ }^{1}$

57. ELEUSINE Gaertn. Goose Grass. Yard Grass

*1. indica Gaertn. ${ }^{1}$

58. LEPTOCHLOA Beauv.

2. fascicularis (Lam.) Gray. Diplaona Beauv. ${ }^{1}$

60. PHRAGMITES Trin. Reed

1. communis Trin. ${ }^{1}$ P. vulgaris (Lam.) B S P. ${ }^{52}$

62. TRIPLASSIS Beauv.

1. purpurea (Walt.) Chapm. ${ }^{1}$ (Sand Grass.)

63. ERAGROSTIS Beauv.

2. capillaris (I.) Nees. ${ }^{1}$

4. pilosa (L.) Beauv. ${ }^{1}$ E. Purshii Schrad. ${ }^{51}$

*5. megastachya (Koeler.) Link. E. major Host. ${ }^{1}$

*6. minor Host. ${ }^{*}$

8. pectinacea (Michx.) Steud. ${ }^{1}$

pectinacea var. spectabilis Gray. ${ }^{1}$

68. DISTICHLIS Raf. Spike Grass. Alkali Grass

1. spicata (L.) Greene. D. maritima Raf. ${ }^{1}$

69. BRIZA L. Quaking Grass

*1. media L. ${ }^{1}$

70. DACTYLIS L. Orchard Grass

*1. glomerata L. ${ }^{1}$

71. POA L. Meadow Grass. Spear Grass

${ }^{*} 1$. annua L. ${ }^{1}$ (Low Spear Grass.)

*3. compressa L. ${ }^{1}$ (Canada Blue Grass. Wire Grass.)

9. triflora Gilib. (Fowl Meadow Grass.) P. serotina Ehrh. ${ }^{1}$

10. pratensis L. ${ }^{1}$ (June Grass, Spear Grass, Kentucky Blue Grass.)

*11. trivialis L. ${ }^{1}$ (Rough-stalked Meadow Grass.)

13. debilis Torr. ${ }^{1}$ 
73. GLYCERIA R. Br. Manna Grass

2. obtusa (Muhl.) Trin. ${ }^{34}$

3. canadensis (Michx.) Trin..$^{34}$ (Rattlesnake Grass.)

5. nervata (Willd.) Trin. ${ }^{1}$ (Fowl Meadow Grass.)

6. grandis Wats. (Reed Meadow Grass.) G. aquatica var. americana Vasey. ${ }^{1}$

7. pallida (Torr.) Trin. ${ }^{1}$

8. fluitans (L.) R. Br. ${ }^{1}$

10. borealis (Nash) Batchelder. ${ }^{3}$

11. acutiflora Torr. ${ }^{1}$

74. PUCCINELLIA Parl.

1. maritima (Huds.) Parl. ${ }^{50}$ (Goose Grass, Sea Spear Grass.) Glyceria R. Br. ${ }^{1}$

3. distans (L.) Parl. Glyceria R. Br. ${ }^{1}$

75. FESTUCA Fescue Grass

*1. myuros L. ${ }^{1}$

3. octoflora Walt. F. tenella Willd. ${ }^{1}$

4. rubra L. ${ }^{1}$

6. ovina L. ${ }^{1}$ (Sheep's Fescue.) ovina var. duriuscula (L.) Koch. F. duriuscula L.'

*7. elatior L. (Taller or Meadow Fescue.) B. U.

8. nutans Spreng. ${ }^{1}$

76. BROMUS L. Brome Grass

*1. secalinus L. ${ }^{1}$ (Cheat or Chess.)

*2. hordeaceus L. (Soft Chess.) B. mollis L..'

*3. racemosus $\mathrm{L}^{\mathrm{t}}$

*8. tectorum L. ${ }^{1}$

*9. sterilis L. ${ }^{1}$

11. ciliatus $\mathrm{L}^{\mathrm{I}}$

12. purgans L. B. ciliatus var. Gray. ${ }^{1}$

77. LOLIUM L. Darnel

*1. perenne L. ${ }^{1}$ (Common D., Perennial Ray or Rye Grass.)

*3. temulentum L. ${ }^{3}$ (Bearded D.)

79. AGROPYRON Gaertn.

*3. repens (L.) Beauv. ${ }^{1}$ (Couch, Quitch or Quick Grass.) repens var. agrestis Anderss. ${ }^{3}$

7. caninum (L.) Beauv. ${ }^{1}$ (Awned Wheat Grass.) 
80. HORDEUM (Tourn L.) Barley

1. jubatum L. ${ }^{3}$ (Squirrel-tail Grass.)

H. distichum L. ${ }^{3}$ (Two-rowed B.)

81. ELYMUS L. Wild Rye. Lyme Grass

1. virginicus $L .{ }^{1}$

3. canadensis $L .{ }^{1}$

E. riparius Wiegand. ${ }^{49}$

6. striatus Willd. ${ }^{1}$

82. HYSTRIX Moench. Bottle-brush Grass

1. patula Moench. Asprella hystrix Schreb. ${ }^{\mathrm{t}}$

Triticum

sativum Lam. ${ }^{3}$

sativum var. muticum Hack. ${ }^{3}$

sativum var. vulgare Hack. ${ }^{3}$

Secale

* cereale L. $^{3} \quad$ (Rye.)

Zea

* mays L. ${ }^{3}$ (Maize, Indian Corn.)

FAMULY 21. CYPERACEAE (Sedge Family)

1. GYPERUS (Tourn.) L. Galingale

1. flavescens L. ${ }^{1}$

2. diandrus Torr. ${ }^{38}$

3. rivularis Kunth..$^{38}$

4. Nuttallii Eddy. ${ }^{38}$

8. aristatus Rottb. ${ }^{38}$ C. inflexus Muhl. ${ }^{1}$

15. dentatus Torr. ${ }^{38}$

18. esculentus L. ${ }^{38}$ C. phymatodes Muhl. ${ }^{1}$ esculentus var. leptostachyus Boeckl. ${ }^{38}$

20. ferax Rich. ${ }^{38}$ C. Michauxianus Schultze. ${ }^{1}$

22. strigosus $\mathrm{L}^{38}$

32. Grayii Torr. ${ }^{38-42}$

34. filiculmis Vahl. ${ }^{38}$

filiculmis var. macilentus Fernald. ${ }^{38}$

3. DULICHIUM Pers.

1. arundinaceum (L.) Britton. ${ }^{34-38}$ D. spathaceum Pers. ${ }^{1}$

4. ELEOCHARIS R. Br. Spike Rush

1. interstincta (Vahl.) R. \& S. ${ }^{38} \quad$ E. equisetoides Torr. ${ }^{1}$ 
3. Robbinsii Oakes. ${ }^{38}$

5. olivacea Torr. ${ }^{38}$

10. obtusa (Willd.) Schultes..$^{38}$ E. avata (Willd.) R. Br. ${ }^{52}$

12. palustris (L.) R. \& S. ${ }^{38}$

13. acicularis (L.) R. \& S. ${ }^{38}$

16. tuberculosa (Michx.) R. \& S. ${ }^{38}$

18. melanocarpa Torr. ${ }^{38}$

21. tenuis (Willd.) Schultes. ${ }^{38}$

26. rostellata Torr. ${ }^{38}$

6. PSILOCARYA Torr. Bald Rush

1. scirpoides Torr. ${ }^{38}$ Rhynchospora Gray. ${ }^{1}$

7. STENOPHYLLUS Raf.

1. capillaris (L.) Britton. ${ }^{38}$ Fimbristylis Gray. ${ }^{34}$

8. FIMBRISTYLIS Vahl.

5. autumnalis (L.) R. \& S. ${ }^{1}$

6. Frankii Steud. ${ }^{38}$

9. SCIRPUS (Tourn.) L. Bulrush or Club Rush

1. nanus Spreng. ${ }^{38}$ Eleocharis pygmaea Torr. ${ }^{1}$

4. planifolius Muhl. ${ }^{38}$

6. hudsonianus (Michx.) Fernald..$^{35-38}$

7. subterminalis Torr. ${ }^{38}$

10. debilis Pursh. ${ }^{38}$

11. Smithii Gray. ${ }^{38}$

12. americanus Pers. ${ }^{38}$ S. pungens Vahl. ${ }^{1}$

13. Torreyi Olney. ${ }^{38}$

14. Olneyi Gray. ${ }^{38}$

17. validus Vahl. ${ }^{38}$ (Great B.)

20. fluviatilis (Torr.) Gray. ${ }^{38}$ (River B.)

21. robustus Pursh. S. maritimus var. macrostachy's.

22. campestris var. paludosus (A. Nelson.) Fernald. ${ }^{38} S$. maritimus L. ${ }^{1}$

campestris var. novae-angliae (Britton.) Fernald. ${ }^{38}$

24. sylvaticus L. ${ }^{38}$

25. atrovirens Muhl. ${ }^{38}$

27. georginaus Harper. ${ }^{38}$

28. polyphyllus Vahl. ${ }^{38}$

32. cyperinus (L.) Kunth. ${ }^{38}$ (Wool Grass.) 
32. cyperinus var. pelius Fernald. ${ }^{38}$

cyperinus var. condensatus Fernald. ${ }^{38}$

33. Eriophorum Michx. ${ }^{1}$

35. atrocinctus Fernald. ${ }^{38}$

10. ERIOPHORUM L. Cotton Grass

2. callitrix Cham. ${ }^{38}$ (Hare's Tail.) E. vaginatum L. ${ }^{27}$

4. gracile Roth. ${ }^{38}$

5. tenellum Nutt. ${ }^{38}$

6. angustifolium Roth. E. polystachion L. ${ }^{1}$

7. viridi-carinatum (Engelm.) Fernald..$^{38}$

8. virginicum L. ${ }^{34-38}$

11. FUIRENA Rottb. Umbrella Grass

1. squarrosa Michx. ${ }^{38}$

12. HEMIGARPHA Nees \& Arn.

1. micrantha (Vahl.) Britton. ${ }^{38}$ H. subsquarrosa Nees. ${ }^{1}$

14. RYNGHOSPORA Vahl. Beak Rush

2. macrostachya Torr. ${ }^{34-38}$

7. fusca (L.) Ait. F. ${ }^{34-38}$

11. alba (L.) Vahl. ${ }^{38}$

14. glomerata (L.) Vahl. ${ }^{38}$

15. CLADIUM P. Br. Twig Rush

1. mariscoides (Muhl.) Torr. ${ }^{38}$

16. SCLERIA Bergius. Nut Rush

1. triglomerata Michx. ${ }^{1}$

6. reticularis $\mathrm{Michx} .^{38}$

reticularis var. pubescens Britton. ${ }^{38}$ S. laxa Torr. ${ }^{1}$

18. GAREX (Ruppius.) L. Sedge

2. scoparia Schkuhr. ${ }^{19}$

scoparia var. condensa Fernald. ${ }^{19}$

3. tribuloides Wahlenb. C. lagopodioides Schkuhr. ${ }^{1}$

4. siccata Dewey. ${ }^{19}$

5. Crawfordii Fernald. C. scoparia var. minor Boott. ${ }^{1}$

8. cristata Schwein. ${ }^{19}$

9. albolutescens Schwein. ${ }^{19}$

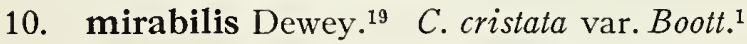
mirabilis var. perlonga Fernald. ${ }^{19}$ 
11. straminea Willd. ${ }^{19}$ C. straminea var. minor. ${ }^{1}$

12. hormathodes Fernald. C. straminea var. aperta Boott. ${ }^{1}$ C. tenera Dewey. ${ }^{19}$

hormathodes var. invisa (W. Boott.) Fernald. C. tenera var. invisa Britton. ${ }^{19}$

14. silicea Olney. ${ }^{19}$

15. alata Torr. ${ }^{19}$

17. festucacea Schkuhr. ${ }^{19}$ C. straminea var. Boott. ${ }^{1}$

festucacea var. brevior (Dewey) Fernald. ${ }^{19}$ C. straminea var. typica. ${ }^{1}$

19. foenea Willd. ${ }^{1}$

foenea var. perplexa Bailey. ${ }^{19}$

23. adusta Boott. ${ }^{1}$

26. exilis Dewey. ${ }^{1-11-19}$

27. stellulata Good. ${ }^{19}$ stellulata var. ormantha Fernald. ${ }^{10}$ stellulata var. excelsior (Bailey) Fernald. ${ }^{19}$ stellulata var. cephalantha (Bailey) Fernald. ${ }^{19}$ C. stellulata L. var. seirpoides. ${ }^{\text {I }}$ stellulata var. angustata Carey. ${ }^{19}$

28. sterilis Willd. ${ }^{19}$

29. scirpoides Schkuhr. C. interior Bailey. ${ }^{19}$ scirpoides var. capillacea (Bailey) Ferna'd. ${ }^{19}$

30. seorsa E. C. Howe. ${ }^{19}$

32. canescens L. ${ }^{1}$ canescens var. disjuncta Fernald. ${ }^{19}$

33. brunnescens Poir. ${ }^{19}$

34. bromoides Schkuhr. ${ }^{19}$

35. Deweyana Shwein. ${ }^{19}$

37. trisperma Dewey. ${ }^{19}$

41. rosea Schkuhr. ${ }^{19}$ rosea var. radiata Dewey. ${ }^{3-19}$

42. retraflexa Muhl. ${ }^{1}$ C. rosea var. Torr. ${ }^{19}$

*43. muricata L. ${ }^{19}$

44. Muhlenbergii Schkuhr. ${ }^{19}$ Muhlenbergii var. enervis Boott. ${ }^{19}$

45. cephalophora Muhl. ${ }^{19}$

47. sparganioides Muhl. ${ }^{19}$

48. cephaloidea Dewey. ${ }^{19}$ 
51. vulpinoidea Michx. ${ }^{19}$

52. setacea Dewey. var. ambigua (Barratt) Fernald. C. xanthocarpa Bicknell. ${ }^{19}$

54. diandra Schrank. C. teretiuscula Good.19-11

56. stipata Muhl. ${ }^{19}$

65. crinita Lam. ${ }^{19}$ C. Mitchelliana M. A. Curtis. ${ }^{1}$ crinita var. minor Boott. ${ }^{19}$

crinita var. gynandra (Schwein.) Schwein. \& Torr. ${ }^{19}$ C. gynandra Schwein. ${ }^{1}$

68. torta Boott. ${ }^{19}$

70. Goodenowii J. Gay. C. vulgaris Fries. ${ }^{1}$

71. stricta Lam. ${ }^{19}$ C. virginiana Sm. ${ }^{1}$ stricta var. angustata (Boott.) Bailey. ${ }^{19}$ stricta var. decora Bailey. ${ }^{19}$ C. strictior Dewey. ${ }^{1}$

72. aurea Nutt. ${ }^{19}$

75. leptalea Wahlenb. ${ }^{19} \quad$ C. polytrichoides Muhl. ${ }^{1}$

80. polygama Schkuhr. C. Buxbaumii Wahlenb. ${ }^{1} \quad$ C. fusca Allioni. ${ }^{19}$

81. triceps Michx. var. hirsuta (Willd.) Bailey. ${ }^{19}$ C. triceps Michx. ${ }^{\mathrm{t}}$

82. virescens Muhl. var. costata Dewey. ${ }^{19}$ virescens var. Swanii Fernald. C. virescens Man. 6 Ed. ${ }^{19}$

85. gracillima Schwein. ${ }^{19}$

93. umbellata Schkuhr. ${ }^{19}$

96. albicans Willd. C. Emmonsii var. elliptica Boott. ${ }^{1}$

97. communis Bailey. C. pilulifera Fernald. ${ }^{19}$

98. varia Muhl. ${ }^{19}$ C.Emmonsii Dewey. ${ }^{1}$

99. novae-angliae Schwein. ${ }^{1}$

100. pennsylvanica Lam. ${ }^{19}$

pennsylvanica var. lucorum (Willd.) Fernald. ${ }^{19}$

101. pubescens Muhl. ${ }^{19}$

105. panicea L. ${ }^{19}$

106. tetanica Schkuhr. var. Meadii (Dewey.) Bailey. ${ }^{19}$

107. polymorpha Muhl. ${ }^{19}$

110. pallescens L. ${ }^{19}$

112. limosa L. ${ }^{1}$

115. prasina Wahlenb. ${ }^{19} \quad$ C. miliacea Muhl. ${ }^{1}$

121. plantaginea Lam. C. laxiflora Lam. var. ${ }^{1}$

123. platyphylla Carey. ${ }^{19}$ 
124. laxiculmis Schwein. ${ }^{19}$ C. retrocuroa Dewey. ${ }^{1}$

125. digitalis Willd. ${ }^{19}$

126. ptychocarpa Steud. ${ }^{19}$

127. laxiflora Lam. ${ }^{19}$ C. laxiflora Lam. var. intermedia. ${ }^{1}$

laxiflora var. patulifolia (Dewey.) Carey. ${ }^{19}$

laxiflora var. varians Bailey. ${ }^{19} \quad$ C. blanda Dewey. ${ }^{1}$

laxiflora var. blanda (Dewey.) Boott. var. striatula Carey. ${ }^{19}$

C. blanda var. major. ${ }^{1}$

131. conoidea Schkuhr. ${ }^{19}$

132. grisea Wahlenb. ${ }^{19}$

136. Crawei Dewey. ${ }^{19}$

138. flava L. ${ }^{1}$

145. arctata Boott. ${ }^{19}$

146. debilis Michx. ${ }^{1}$

debilis var. Rudgei Bailey. ${ }^{19}$

150. scabrata Schwein. ${ }^{19}$

151. filiformis L. ${ }^{19}$

152. lanuginosa Michx. ${ }^{1}$ C. filiformis var. latifolia Boeckl. ${ }^{19}$

154. vestita Willd. ${ }^{19}$

155. striata Michx. var. brevis Bailey. ${ }^{19}$ C. striata Michx. ${ }^{1}$

159. riparia W. Curtis. ${ }^{19}$

161. squarrosa L. ${ }^{19}$

164. Pseudo-Cyperus L. ${ }^{1}$

165. comosa Boott. C. Pseudo-Cyperus var. Boott. ${ }^{1}$

166. hystericina Muhl. ${ }^{19}$

167. Lurida Wahlenb. ${ }^{19}$ C. tentaculata Muhl. ${ }^{1}$

lurida var. exudans Bailey. ${ }^{19}$

169. retrorsa Schwein. ${ }^{19}$

172. lupuliformis Sartwell. ${ }^{1}$ C. lupulina var. polystachya S. \& T. ${ }^{19}$

173. lupulina Muhl. ${ }^{19}$

lupulina var. pedunculata Dewey. ${ }^{3-19}$

175. intumescens Rudge. ${ }^{19}$

176. folliculata L. ${ }^{3-19}$

178. subulata Michx. ${ }^{19}$

182. vesicaria $L .{ }^{19}$

vesicaria var. monile (Tuckerm.) Fernald. ${ }^{19}$ C. monile Tuckerm. ${ }^{1}$ 
182. vesicaria var. jejuna Fernald. ${ }^{19}$ C. monile var. minor Olney. ${ }^{1}$

183. rostrata Stokes. ${ }^{19}$ C. utriculata var. minor Boott. ${ }^{1}$ rostrata var. utriculata (Boott.) Bailey. C. utriculata Boott. ${ }^{1}$

184. bullata Schkuhr. ${ }^{19}$ C. Olneyi Boott. ${ }^{1}$ bullata var. Olneyi Fernald. ${ }^{19}$

185. Tuckermani Dewey. ${ }^{19}$

FAMILY 22. ARACEAE (Arum Family)

$$
\text { ORDER VIII ARALES }
$$

1. ARISAEMA Martius. Indian Turnip. Dragon Arum

1. triphyllum (L.) Schott. ${ }^{34}$ (Indian Turnip, Jack-in-thePulpit.)

2. Dracontium (L.) Schott. ${ }^{1}$ (Green Dragon, Dragon Root.)

2. PELTANDRA Raf. Arrow Arum

1. virginica (L.) Kunth. ${ }^{1}$

2. CALLA L. Water Arum

1. palustris $L^{1}$

4. SYMPLOGARPUS Salisb. Skunk Cabbage

1. foetidus (L.) Nutt. ${ }^{1}$

5. ORONTIUM L. Golden Club

1. aquaticum L. ${ }^{34}$

6. ACORUS L. Sweet Flag. Calamus

1. calamus L. ${ }^{1}$

FAMILY 23. LEMNACEAE (Duckweed Family)

1. SPIRODELA Schleid.

1. polyrhiza (L.) Schleid. Lemna L. ${ }^{1}$

2. LEMNA L. Duckweed. Duck's-meat

2. valdiviana Philippi. $^{3}$

4. minor L. ${ }^{\mathrm{I}}$

ORDER IX XIRIDALES

FAMILY 24. ERIOGAULACEAE (Pipewort Family)

1. ERIOCAULON (Gronov.) L. Pipewort

3. articulatum (Huds.) Morong. E. septangulare With..$^{20-34}$ 
FAMILY 25. XYRIDACEAE (Yellow-eyed Grass Family)

1. XYRIS (Gronov.) L. Yellow-eyed Grass

1. montana Ries. $X$. flexuosa Willd. ${ }^{34}$

2. caroliniana Walt. ${ }^{34}$

FAMILY 27. COMMELINACEAE (Spiderwort Family)

2. COMMELINA (Plum.) L. Day Flower

1. communis L. Miss E. M. Round.

4. virginica $L . .^{2}$

FAMILY 29. PONTEDERIACEAE (Pickerel-weed Family)

1. PONTEDERIA L. Pickerel-weed

1. cordata $\mathrm{L}^{34}$

cordata var. lancifolia Morong. ${ }^{34}$

ORDER X LILIALES

FAMILY 30. JUNCACEAE (Rush Family)

1. JUNCUS (Tourn.) L. Rush. Bog Rush

1. bufonius L.22

3. Gerardi Loisel..22 (Black Grass.)

4. tenuis Willd.2 tenuis var. Williamsii Fernald.22-34

7. secundus Beauv. ${ }^{22}$ J. temuis var. Engelm. ${ }^{3}$

8. Dudleyi Wiegand. ${ }^{22}$

9. dichotomus Ell. ${ }^{44}$ dichotomus var. platyphyllus IViegand ${ }^{41}$

13. Greenei Oakes \& Tuckerm. ${ }^{22}$

14. balticus Willd. var. littoralis Engelm. J.Balticus Dethard. ${ }^{1}$

16. effusus L.22

23. brevicaudatus (Engelm.) Fernald. ${ }^{22-34}$

24. canadensis J. Gay. ${ }^{22-34}$ J. canadensis var. longicaudatus Engelm. ${ }^{1-52}$

canadensis var. subcaudatus Engelm. ${ }^{1}$

25. pelocarpus Mey..$^{22-34}$

28. militaris Bigel. ${ }^{22-34}$

30. nodosus $L .{ }^{1}$

33. scirpoides Lam. ${ }^{1}$

35. acuminatus Michx. ${ }^{22}$ 
36. debilis Gray. ${ }^{32} \quad J$. acuminatus var. Engelm.

40. articulatus $L .{ }^{22}$

articulatus var. obtusatus Engelm..$^{22}$

43. marginatus Rostk. ${ }^{22-34}$

marginatus var. paucicapitatus Engelm. ${ }^{3}$

2. LUZULA D C. Wood Rush

1. saltuensis Fernald. L. pilosa Willd. ${ }^{1}$

6. campestris (L.) D. C. var. multiflora (Ehrh.) Celak. ${ }^{22}$ L. campestris D C. ${ }^{1}$

FAMILY 31. LILIACEAE (Lily Family)

9. MELANTHIUM L.

1. virginicum L. ${ }^{1}$ (Bunch-flower.)

10. VERATRUM (Tourn.) L. False Hellebore

1. viride Ait. ${ }^{1}$ (American White Hellebore, Indian Poke.)

11. UVULARIA L. Bellwort

1. perfoliata L. ${ }^{1}$

12. OAKESIA Wats.

1. sessilifolia (L.) Wats. Uvularia L.1

13. ALLIUM (Tourn.) L. Onion. Garlic

1. tricoccum Ait. ${ }^{\mathrm{I}}$ (Wild Leek.)

6. canadense L. ${ }^{1}$ (Wild Garlic.)

*8. vineale L. ${ }^{1} \quad$ (Field Garlic.)

Funkia

* F. ovata Spreng. (Blue Day Lily.) ${ }^{2-37}$

15. HEMEROCALLIS L. Day Lily

*1. fulva L. ${ }^{1}$ (Common D.)

16. LILIUM (Tourn.) L. Lily

1. philadelphicum L. ${ }^{1}$ (Wild Orange-red L., Wood L.)

3. superbum L. ${ }^{1}$ (Turk's-cap L.)

5. canadense L. ${ }^{1}$ (Wild Yellow L.)

*7. tigrinum Ker. ${ }^{30}$ (Tiger L.)

17. ERYTHRONIUM L. Dog's-tooth Violet

1. americanum Ker. ${ }^{1}$ (Yellow Adder's-tongue.)

19. ORNITHOGALUM (Tourn. L.) Star of Bethlehem.

*1. umbellatum L. ${ }^{30}$ 
22. ASPARAGUS (Tourn.) L. Asparagus

*1. officinalis L. ${ }^{1}$ (Garden A.)

23. CLINTONIA Raf.

1. borealis (Ait.) Raf. ${ }^{1}$

24. SMILACINA Desf. False Solomon's Seal

1. racemosa (L.) Desf. ${ }^{1}$ (False Spikenard.)

2. stellata (L.) Desf. ${ }^{1}$

3. trifolia (L.) Desf. ${ }^{1}$

25. MAIANTHEMUM (Weber in) Wiggers.

1. canadense Desf. Smilacina bifolia var. canadensis Gray. ${ }^{1}$

28. POLYGONATUM (Tourn.) Hill. Solomon's Seal

1. biflorum (Walt.) Ell. ${ }^{1}$ (Small S.)

2. commutatum (R.\&S.) Dietr. (GreatS.) P. giganteum Dietr. $^{1}$

30. MEDEOLA (Gronov.) L. Indian Cucumber-root 1. virginiana $L .{ }^{34}$

31. TRILLIUM L. Wake Robin. Birthroot

4. erectum L. ${ }^{1}$

5. grandiflorum (Michx.) Salisb. ${ }^{16}$

6. cernuum L. ${ }^{34}$

9. undulatum Willd. ${ }^{34}$ (Painted T.) T. erythrocarpum Michx. ${ }^{30}$

32. ALETRIS L. Colic-root. Star Grass

1. farinosa L. ${ }^{1}$

33. SMILAX (Tourn.) L. Green Brier. Cat Brier

1. herbacea L. ${ }^{1}$ (Carrion-flower.)

5. rotundifolia L. ${ }^{1}$ (Common Green Brier, Horse Brier.) rotundifolia var. quadrangularis (Muhl.) Wood. ${ }^{1}$

6. glauca Walt. ${ }^{1}$ (Saw Brier.)

FAMILY 32. HAEMODORACEAE (Bloodwort Family)

1. LACHNANTHES Ell. Red-root

1. tinctoria (Walt.) Ell. ${ }^{1}$

L. capitata ${ }^{34}$

FAMILY 33. DIOSCOREACEAE (Yam Family)

1. DIOSCOREA (Plumier) L. Yam

1. villosa L. ${ }^{1}$ (Wild Yam-root) 
FAMILY 34. AMAR YLLIDACEAE (Amaryllis Family)

6. HYPOXIS L. Star Grass

1. hirsuta (L.) Coville. H. erecta L. ${ }^{34}$

FAMILY 35. IRIDACEAE (Iris Family)

1. IRIS (Tourn.) L. Fleur-de-lis

1. versicolor L. ${ }^{1}$ (Larger Blue Flag.)

4. prismatica Pursh. ${ }^{37}$ (Slender Blue Flag.) I. virginica L. ${ }^{1}$

4. SISYRINCHIUM L. Blue-eyed Grass

6. angustifolium Mill..$^{3-37}$

11. gramineum Curtis. ${ }^{41}$ S. anceps Cav. ${ }^{1}$

12. atlanticum Bicknell. ${ }^{41}$

ORDER XII ORCHIDALES

FAMILY 38. ORCHIDACEAE (Orchis Family)

1. CYPRIPEDIUM L. Lady's Slipper. Mocassin

Flower

2. parviflorum Salisb. ${ }^{1}$ (Smaller Yellow L.) parviflorum var. pubescens (Willd.) Knight. (Larger

Yellow L.) C. pubescens Willd. ${ }^{15}$

6. acaule Ait. ${ }^{9-15-34}$ (Stemless L.)

2. ORCHIS (Tourn.) L.

2. spectabilis L. ${ }^{15}$ (Showy O.)

3. HABENARIA Willd. Rein Orchis. Fringed Orchis

1. bracteata (Willd.) R. Br. ${ }^{15} \quad$ H. viridis var. Reich.

2. flava (L.) Gray. H. virescens Spreng..$^{15}$

7. clavellata (Michx.) Spreng. ${ }^{34}$ H. tridentata Hook. ${ }^{1}$

10. Hookeri Torr. ${ }^{1}$ H. Hookeriana Gray. ${ }^{15}$

14. ciliaris (L.) R. Br. ${ }^{15-30}$ (Yellow Fringed O.)

15. blephariglottis (Willd.) Torr. ${ }^{15-30-34}$ (White Fringed O.) blephariglottis var. holopetala (Lindl.) Gray. ${ }^{1}$

16. lacera (Michx.) R. Br. ${ }^{15}$ (Ragged Fringed O.)

18. psycodes (L.) Sw. ${ }^{15-34}$

19. fimbriata (Ait.) R. Br. ${ }^{15}$

4. POGONIA Juss.

1. ophioglossoides (L.) Ker. ${ }^{15}$

2. trianthophora (Sw.) B S P. P. pendula Lindl. ${ }^{1}$

4. verticillata (Wild.) Nutt. ${ }^{15}$ 
5. CALOPOGON R. Br.

1. pulchellus (Sw.) R. Br. ${ }^{15}$

6. ARETHUSA (Gronov.) L.

1. bulbosa L. ${ }^{15}$

8. SPIRANTHES Richard. Ladies' Tresses

1. Beckii Lindl. S. simplex Gray. ${ }^{15}$

2. gracilis (Bigel.) Beck..$^{15-34}$

4. praecox (Walt.) Wats. \& Coult. ${ }^{15}$ S. graminea Lindl. var. Walteri. ${ }^{1}$

7. cernua (L.) Richard. ${ }^{15}$

9. Romanzoffiana Cham.

9. EPIPACTIS (Haller.) Boehm. Rattlesnake Plantain

1. repens (L.) Crantz. Goodyear $\mathrm{R} . \mathrm{Br}^{1}{ }^{1}$

4. pubescens (Willd.) A. A. Eaton. Goodyear R. Br. ${ }^{15-34}$

10. LISTERA R. Br. Twayblade

1. cordata (L.) R. Br. ${ }^{15}$

11. CORALLORRHIZA (Haller.) R. Br. Coral Root

1. trifida Chatelain. C. innata R. Br. ${ }^{21}$

2. maculata Raf. C. multiflora Nutt. ${ }^{15-21}$

4. odontorhiza Nutt. ${ }^{15-21}$

13. MICROSTYLIS (Nutt.) Eaton. Adder's Mouth

2. unifolia (Michx.) B S P. M. ophioglossoides Nutt. ${ }^{15-30}$

14. LIPARIS Richard. Twayblade

1. liliifolia (L.) Richard. ${ }^{1}$

2. Loeselii (L.) Richard. ${ }^{15}$

\section{Class II Dicotyledoneae \\ Subclass I Archichlamydeae \\ ORDER XIII PIPERALES}

FAMILY 39. PIPERACEAE (Pepper Family)

1. SAURURUS (Plum.) L. Lizard's Tail

1. cernuus L. ${ }^{25-26}$

ORDER XIV SALICALES

FAMILY 40. SALICACEAE (Willow Family)

1. SALIX (Tourn.) L. Willow. Osier 
1. nigra Marsh. ${ }^{1}$ (Black W.) nigra var. falcata (Pursh.) Torr. ${ }^{1}$

3. amygdaloides Anders. (Peach-leaved W.) S. nigra var.'

5. lucida Muhl. ${ }^{1}$ (Shining W.)

*7. fragilis L. ${ }^{1}$ (Crack W.)

*8. alba L. ${ }^{1}$ (White W.)

*9. babylonica L. (Weeping W.)

10. longifolia Muhl. ${ }^{1}$ (Sand Bar W.)

11. cordata Muhl. ${ }^{1}$

16. pedicellaris Pursh. S. myrtilloides L.'

19. discolor Muhl. ${ }^{1}$ (Glaucous W.)

20. petiolaris $\mathrm{Sm} .^{1}$

21. humilis Marsh. ${ }^{1}$ (Prairie W.)

22. tristis Ait. ${ }^{1}$ (Dwarf Gray W.)

23. sericea Marsh. ${ }^{1}$ (Silky W.)

24. rostrata Richards. S. livida var. occidentalis Gray. ${ }^{1}$

*28. viminalis L. ${ }^{\mathrm{I}}$ (Osier.)

*31. purpurea L. ${ }^{1}$ (Purple W.)

2. POPULUS (Tourn.) L. Poplar. Aspen

*1. alba L. ${ }^{1}$ (White, P. Silver-leaved P., Abele.)

2. tremuloides Michx. ${ }^{1}$ (American A.)

3. grandidentata Michx. ${ }^{1}$ (Large-toothed A.)

*6. candicans Ait. (Balm of Gilead.) P. balsamifera var. Gray. ${ }^{1}$

7. deltoides Marsh. (Cottonwood, Necklace P.) P. monilifera Ait. ${ }^{1}$

ORDER XV MYRICALES

FAMILY 41. MYRIGACEAE (Sweet Gale Family) 1. MYRICA L.

1. Gale L. ${ }^{1}$ (Sweet Gale.)

2. cerifera L.I (Wax Myrtle.)

4. asplenifolia L. ${ }^{34}$ (Sweet Fern.) Comptonia Ait. ${ }^{1}$

ORDER XVII JUGLANDALES

FAMILY 43. JUGLANDAGEAE (Walnut Family)

1. JUGLANS L. Walnut

1. cinerea L. ${ }^{1}$ (Butternut, White W.)

2. nigra L.' (Black W.) 
2. CARYA Nutt. Hickory

2. ovata (Mill.) K. Koch. (Shell-bark or Shag-bark H.) C. alba Nutt.

3. laciniosa (Michx. f.) Loud. (Big Shell-bark, King Nut.) C. sulcata Nutt. ${ }^{1}$

4. alba (L.) K. Koch. (Mocker Nut, White-heart H.) C. tomentosa Nutt. ${ }^{1}$

5. microcarpa Nutt. ${ }^{1}$

7. glabra (Mill.) Spach. (Pignut or Broom H.) C. porcina Nutt. ${ }^{1}$

8. cordiformis (Wang.) K. Koch. (Bitter Nut or Swamp H.) C. amara Nutt. ${ }^{1}$

\section{ORDER XVIII FAGALES}

FAMILY 44. BETULACEAE (Birch Family)

1. CORYLUS (Tourn.) L. Hazelnut. Filbert

1. americana Walt. ${ }^{\mathrm{I}}$ (Hazelnut.)

2. rostrata Ait. ${ }^{1}$ (Beaked H.)

2. OSTRYA (Mich.) Scop. Hop Hornbeam. Ironwood 1. virginiana (Mill.) K. Koch. (American Hop H., Leverwood.) O. virginica Willd. ${ }^{1}$

3. CARPINUS (Tourn.) L. Hornbeam. Ironwood

1. caroliniana Walt. (American H., Blue or Water Beech.) C. americana Michx. ${ }^{1}$

4. BETULA (Tourn.) L. Birch

1. lenta L. ${ }^{1}$ (Cherry, Sweet, or Black Birch.)

2. lutea Michx. f. ${ }^{1}$ (Yellow or Gray B.)

3. nigra L. ${ }^{1}$ (River or Red B.)

4. populifolia Marsh. (White, Gray or Old Field B.) B. alba var. Spach. ${ }^{1}$

6. alba L. (Paper, Canoe or White B.) alba var. minor (Tuckerm.) Fernald. B. papyracea Ait.1

5. ALNUS (Tourn.) Ell. Alder

3. incana (L.) Moench. ${ }^{1}$ (Speckled or Hoary A.)

4. rugosa (Du Roi) Spreng. (Smooth A.) A. serrulata Willd. ${ }^{1}$ 
FAMILY 45. FAGACEAE (Beech Family)

1. FAGUS (Tourn.) L. Beech

1. grandifolia Ehrh. F. ferruginea Ait. ${ }^{1}$

2. GASTANEA (Tourn.) Hill. Chestnut

1. dentata (Marshl.) Borkh. (Chestnut.) C. vesca L. var. americana Michx. ${ }^{1}$

3. QUERCUS (Tourn.) L. Oak

1. alba L. ${ }^{1}$ (White O.)

2. stellata Wang. (Post O., Iron O.) Q. obtusiloba Michx. ${ }^{1}$

4. macrocarpa Michx. ${ }^{1}$ (Bur O., Over-cup or Mossy-cup O.)

5. bicolor Willd. ${ }^{1}$ (Swamp White O.)

8. prinoides Willd. Q. prinus var. humilis Marsh. ${ }^{1}$

9. prinus L. ${ }^{1}$ (Chestnut O.) Q. primus var. monticola Michx. ${ }^{1}$

11. rubra L. ${ }^{1}$ (Red O.)

rubra var. ambigua (Michx. f.) Fernald. Coccinea var. ambigua Gray. ${ }^{1}$

12. palustris Moench. ${ }^{1}$ (Swamp Spanish or Pin O.)

13. coccinea Moench. ${ }^{1}$ (Scarlet O.)

16. velutina Lam. (Quercitron, Yellow-barked or Black O.)

Q. coccinea var. tinctoria A. D C. ${ }^{1}$

17. falcata Michx. Q. rubra var. runcinata A. D C. ${ }^{1}$

18. ilicifolia Wang. ${ }^{1}$ (Bear or Black Scrub Oak.)

ORDER XIX URTICALES

FAMILY 46. URTICACEAE (Nettle Family)

1. ULMUS (Tourn.) L. Elm

1. fulva Michx. ${ }^{1}$ (Slippery or Red E.)

3. americana L. ${ }^{1}$ (American or White E.)

3. CELTIS (Tourn.) L. Nettle-tree. Hackberry

1. occidentalis L. ${ }^{1}$ (Sugarberry.)

4. CANNABIS (Tourn.) L. Hemp

*1. sativa L. ${ }^{1}$

5. HUMULUS L. Hop

1. Lupulus L. ${ }^{1}$ (Common H.) 
8. MORUS (Tourn.) L. Mulberry

*2. alba L. ${ }^{3}$ (White M.)

9. URTICA (Tourn.) L. Nettle

1. gracilis Ait. ${ }^{1}$

*3. dioica L. ${ }^{1}$ (Stinging N.)

*4. urens L. ${ }^{1}$

10. LAPORTEA Gaud. Wood Nettle

1. canadensis (L.) Gaud. ${ }^{1}$

11. PILEA Lindl. Richweed. Clearweed

1. pumila (L.) Gray. ${ }^{1}$

12. BOEHMERIA Jacq. False Nettle

1. cylindrica (L.) $\mathrm{Sw}^{.}{ }^{34}$

13. PARIETARIA (Tourn.) L. Pellitory

1. pennsylvanica Muhl. ${ }^{1}$

ORDER XX SANTALALES

FAMILY 47. SANTALACEAE (Sandalwood Family)

1. COMANDRA Nutt. Bastard Toad-flax

1. umbellata (L.) Nutt. ${ }^{15}$

FAMILY 48. LORANTHACEAE (Mistletoe Family)

2. ARGEUTHOBIUM Bieb.

1. pusillum Peck. ${ }^{27-36}$ (Dwarf Mistletoe.)

\section{ORDER XXII POLYGONALES}

FAMILY 50. POLYGONACEAE (Buckwheat Family)

3. RUMEX L. Dock. Sorrel

*2. patientia L. ${ }^{37}$ (Patience D.)

4. Britannica L. ${ }^{18}$ (Great Water D.)

*5. crispus L. ${ }^{18}$ (Yellow D.)

8. mexicanus Meisn. R. Salicifolius Weinm. ${ }^{18}$

9. al tissimus Wood. ${ }^{2-18}$ (Pale D.)

10. verticillatus $L^{18} \quad$ (Swamp D.)

*12. obtusifolius L. ${ }^{18}$ (Bitter D.)

14. persicarioides L. ${ }^{18}$ (Golden D.) R. maritimus L. ${ }^{1}$

*17. Acetosella L. ${ }^{18}$ (Field or Sheep Sorrel.) 
4. POLYGONUM (Tourn.) L. Knotweed

1. maritimum L. ${ }^{18}$

3. exsertum Small. ${ }^{18}$

4. prolificum (Small.) Robinson. ${ }^{18}$

5. aviculare L. ${ }^{18}$

aviculare var. littorale (Link.) Koch. ${ }^{18}$

6. erectum L. ${ }^{18}$

7. ramosissimum Michx. ${ }^{1}$

ramosissimum forma atlanticum Robinson. ${ }^{18}$

8. tenue Michx. ${ }^{18}$

11. lapathifolium L. ${ }^{18} \quad P$. incarnatum Ell. ${ }^{1}$

14. amphibium L. ${ }^{18}$

amphibium var. Hartwrightii (Gray.) Bissell. ${ }^{18}$ P. Hartwrightii Gray. ${ }^{1}$

15. Muhlenbergii (Meisn.) Wats. ${ }^{18}$

16. pennsylvanicum L. ${ }^{18-34}$

18. Careyi Olney. ${ }^{18-34}$

19. Hydropiper L.18 (Common Smartweed or Water Pepper)

20. acre H B K. ${ }^{18-34}$ (Water Smartweed.)

acre var. leptostachyum Meisn. ${ }^{18}$

*21. orientale L. ${ }^{18}$ (Prince's Feather.)

*22. persicaria L. ${ }^{18-34}$ (Lady's Thumb.)

24. hydropiperoides Michx. ${ }^{18-34}$ (Mild Water Pepper.)

25. virginianum $\mathrm{L}^{16-18}$

26. arifolium L. ${ }^{18-34}$ (Halberd-leaved Tear-thumb.)

27. sagittatum L. ${ }^{18-34}$ (Arrow-leaved Tear-thumb.)

*28. convolvulus L. ${ }^{18}$ (Black Bindweed.)

29. cilinode Michx. ${ }^{18}$

30. scandens L. ${ }^{18}$ (Climbing False Buckwheat.) P. dumetorum var. Gray. ${ }^{34}$ P. dumetorum L. ${ }^{1}$

31. dumetorum L. forma cristatum (Engelm. \& Gray.) Robinson. P. cristatum Engelm. \& Gray. ${ }^{28}$

5. FAGOPYRUM (Tourn.) L. Buckwheat

*1. esculentum Moench. ${ }^{18}$ (Buckwheat.)

6. POLYGONELLA Michx.

1. articulata (L.) Meisn. ${ }^{18}$ Polygonum articulatum L. ${ }^{1}$ 
ORDER XXIII CHENOPODIALES

FAMILY 51. CHENOPODIACEAE (Goosefoot Family)

4. GHENOPODIUM (Tourn.) L. Goosefoot. Pigweed *1. ambrosioides L. ${ }^{2}$ (Mexican Tea.) ambrosioides var. anthelminticum (L.) Gray. ${ }^{1}$ (Wormseed.)

*3. Botrys L. ${ }^{1}$ (Jerusalem Oak, Feather Geranium.)

4. capitatum (L.) Asch. (Strawberry Blite.)

*5. Bonus-Henricus L. ${ }^{2}$ (Good-King-Henry.)

6. rubrum L. (Coast Blite.) Blitum maritimum Nutt. ${ }^{1}$

*8. glaucum L. ${ }^{1}$ (Oak-leaved G.)

9. hybridum L. ${ }^{1}$ (Maple-leaved G.)

*11. album L. ${ }^{1}$ (Lamb's Quarters, Pigweed.) album var. viride (L.) Moq. ${ }^{1}$

*13. murale L. ${ }^{1}$

*14. urbicum L. ${ }^{1}$

5. ATRIPLEX (Tourn.) L. Orach

2. patula L. ${ }^{1}$ patula var. hastata (L.) Gray. ${ }^{3}$

3. arenaria Nutt. ${ }^{1}$

8. SALICORNIA (Tourn.) L. Glasswort. Samphire 1. mucronata Bigel. S. virginica L. ${ }^{1}$

2. europaea L. S. herbacea L. ${ }^{1}$

4. ambigua Michx. S. fruticosa L. var. ${ }^{1}$

9. SUAEDA Forskål. Sea Blite

1. maritima (L.) Dumort. ${ }^{1}$

4. linearis (E U.) Moq. ${ }^{48}$

10. SALSOLA L. Saltwort

1. Kali L. ${ }^{1}$ (Common S.)

* Kali var. tenuifolia G. F. W. Mey. (Russian Thistle.) S. Tragus L. ${ }^{2}$

FAMILY 52. AMARANTHACEAE (Amaranth Family)

1. AMARANTHUS (Tourn.) L. Amaranth

*2. retroflexus L. ${ }^{2}$ (Green A., Pigweed.)

*3. hybridus L. ${ }^{1}$ (Green A., Pigweed.) A. chlorostachys Willd. ${ }^{35}$ 
*3 hybridus forma hypochondriacus (L.) Robinson. A. hypochondriacus L. ${ }^{1}$

*4. paniculatus L. ${ }^{1}$ (Purple A.)

5. graecizans L. (Tumble Weed.) A. albus L.1

6. blitoides Wats. ${ }^{2}$

*7. spinosus L. ${ }^{1}$ (Thorny A.)

10. pumilus Raf. ${ }^{1}$

2. ACNIDA L. Water Hemp

1. cannabina L. ${ }^{1}$

FAMILY 53. PHYTOLACCACEAE (Pokeweed Family)

1. PHYTOLACCA (Tourn.) L. Pokeweed

1. decandra L. ${ }^{20}$ (Common P., Garget, Pigeon Berry.)

FAMILY 55. ILLECEBRACEAE (Knotwort Family)

1. SCLERANTHUS L. Knawel

*1. annuus L. ${ }^{20}$

2. ANYCHIA Michx. Forked Chickweed

1. polygonoides Raf. A. dichotoma Man. ed. $6^{20}$

2. canadensis (L.) B S P. A. capillacea D C. ${ }^{20}$

FAMILY 56. AZOACEAE

2. MOLLUGO L. Indian Chickweed

*1. verticillata $\mathrm{L}^{20}$ (Carpet Weed.)

ORDER XXIV CARYOPHYLLALES

FAMILY 57. GARYOPHYLLACEAE (Pink Family)

1. SPERGULARIA J. \& C. Presl. Sand Spurrey

1. rubra (L.) J. \& C. Presl. ${ }^{20}$ Lepigonium Fries. ${ }^{1}$

2. marina (L.) Griseb. S. salina J.\& C. Presl. ${ }^{20}$ Lepigonium salinum Fries. ${ }^{1}$

3. canadensis (Pers.) Don. S. borealis Robinson. ${ }^{20}$

4. media (L.) C. Presl. Lepigonium medium Fries. ${ }^{1}$

2. SPERGULA L. Spurrey

*1. arvensis L. ${ }^{20-34}$ (Corn S.)

3. SAGINA L. Pearlwort

1. decumbens (Ell.) T. \& G. S. apetala L. ${ }^{1}$

2. procumbens $\mathrm{L}^{20}$ 


\section{ARENARIA L. Sandwort}

1. lateriflora L. ${ }^{20}$

3. peploides L. ${ }^{20}$

*4. serphyllifolia L. ${ }^{20}$ (Thyme-leaved S.)

10. caroliniana Walt. ${ }^{43}$ (Pine-barren S.) A. squarrosa Michx.

11. groenlandica (Retz.) Spreng. ${ }^{20}$ (Mountain S.)

5. STELlaRIA L. Chickweed. Starwort

1. borealis Bigel. ${ }^{20}$

8. longifolia Muhl. ${ }^{20}$

*9. graminea L. ${ }^{20}$

11. pubera Michx. (Great C.)

*12. media (L.) Cyrill. ${ }^{20}$ (Common C.) Alsine L. ${ }^{52}$

6. CERASTIUM L. Mouse-ear Chickweed

1. arvense L..$^{0-37}$ (Field M.)

*2. vulgatum L. ${ }^{20}$ (Common M.)

*3. viscosum $\mathrm{L}^{20}$

*4. semidecandrum L. ${ }^{41}$

8. AGROSTEMMA L. Corn Cockle

*1. Githago L. ${ }^{20}$ Lychnis Sibith ${ }^{51}$

9. LYCHNIS (Tourn.) L. Campion

*2. Flos-cuculi L. ${ }^{2-20}$ (Ragged Robin.)

*4. dioica L. ${ }^{20}$ (Red C.) L. diruna L. ${ }^{1}$

*5. alba Mill. ${ }^{20-34}$ (White C.) L. vespertina Sibth. ${ }^{1}$

10. SILENE Catchfly. Campion

2. antirrhina L. ${ }^{20}$ (Sleepy Catchfly.) antirrhina var. divaricata Robinson..$^{20}$

3. armeria L. ${ }^{2-20}$ (Sweet William Catchfly.)

*6. noctiflora L. ${ }^{20}$ (Night-flowering Catchfly.)

9. pennsylvanica Michx. ${ }^{20}$ (Wild Pink.)

13. stellata (L.) Ait. f. ${ }^{20}$ (Starry Campion.)

*15. latifolia (Mill.) Britten \& Rendle. (Bladder Campion.)

S. inflata Sm. ${ }^{1}$ S. cucubalus Willd. ${ }^{20}$

11. SAPONARIA L.

*1. officinalis L. ${ }^{20}$ (Soapwort, Bouncing Bet.)

*2. Vaccaria L. ${ }^{2-20}$ (Cow-herb.) Vaccaria vulgaris Host. ${ }^{1}$ 
12. GYPSOPHILA L.

*1. muralis $\mathrm{L}^{20}$

14. DIANTHUS L. Pink. Carnation

*4. Armeria L. ${ }^{20}$ (Deptford P.)

FAMILY 58. PORTULACACEAE (Purslane Family)

2. CLAYTONIA (Gronov.) L. Spring Beauty

1. virginica $L .{ }^{20}$

4. PORTUlaCGA (Tourn.) L. Purslane

*1. oleracea L. ${ }^{20}$ (Common P.)

ORDER XXV RANUNCULALES

FAMILY 59. CERATOPHYLLACEAE (Hornwort Family)

1. GERATOPHYLLUM L. Hornwort

1. demersum L. ${ }^{1}$

demersum var. echinatum Gray. ${ }^{1}$

FAMILY 60. NYMPHAEACEAE (Water Lily Family)

1. NYMPHAEA (Tourn.) L. Yellow Pond Lily. Spatterdock

1. advena Ait. (Cow Lily.) Nuphar Ait. f. ${ }^{34}$

x? rubrodisca (Morong.) Greene. N. odorata var. minor. ${ }^{1}$

2. CASTALIA Salisb. Water Nymph. Water Lily

1. odorata (Ait.) Woodville \& Wood. (Sweet-scented Water Lily.) Nymphaea Ait. ${ }^{34}$

odorata forma Rosea (Pursh.) Britton. Nymphaea var. rosea. ${ }^{1}$

4. BRASENIA Schreb. Water Shield

1. Scheberi Gmel. B. peltata Pursh. ${ }^{1}$

FAMILY 61. RANUNCULACEAE (Crowfoot Family)

1. RANUNCULUS (Tourn.) L. Crowfoot. Buttercup

3. aquatilis L. var. capillaceus D C. ${ }^{47}$ (Common White Water C.) R. aquatilis var. trichophyllus Gray. ${ }^{1}$

5. Cymbalaria Pursh. ${ }^{47}$ (Seaside C.)

6. delphinifolius Torr. ${ }^{47}$ (Yellow Water C.) R. multifidus Pursh. ${ }^{1}$

9. laxicaulis (T. \& G.) Darby. ${ }^{47}$ (Water Plantain Spearwort.) $R$. alismaefolius Geyer. ${ }^{1}$ 
10. Flammula L. var. reptans (L.) Mey. ${ }^{1}$

14. sceleratus L. ${ }^{47}$ (Cursed C.)

15. micranthus Nutt. ${ }^{47}$

16. abortivus L. ${ }^{47}$ (Small-flowered C.)

17. allegheniensis Britton. ${ }^{47}$

19. recurvatus Poir. ${ }^{47}$ (Hooked C.)

20. fascicularis Muhl. ${ }^{1}$ (Early C.)

23. repens $L^{47}$ (Creeping $B$.

25. pennsylvanicus L. f. ${ }^{1}$ (Bristly C.)

*27. bulbosus L. ${ }^{47}$ (Bulbous C. or B.)

*28. acris L. ${ }^{47}$ (Tall C. or B.)

5. THALIGTRUM (Tourn.) L. Meadow Rue

5. dioicum L. ${ }^{47}$ (Early M.)

7. revolutum D C. ${ }^{47} T$. purpurascens $\mathrm{L} .{ }^{1}$

8. polygamum Muhl. ${ }^{47}$ (Tall M.) T. cornuti L. ${ }^{1}$

6. ANEMONELLA Spach.

1. thalictroides (L.) Spach. ${ }^{47}$ (Rue Anemone.)

7. HEPATICA (Rupp.) Hill. Liverleaf. Hepatica

1. triloba Chaix. Anemone Hepatica L. var. obtusiloba. ${ }^{1}$

8. ANEMONE (Tourn.) L. Anemone

5. cylindrica Gray. ${ }^{47}$

7. virginiana $L .{ }^{47}$

9. quinquefolia L. ${ }^{47}$ (Wood A.) A. nemorosa L.'

9. CLEMATIS L. Virgin's Bower

1. virginiana $L .{ }^{47}$

11. verticillaris D C. ${ }^{47}$

11. CALTHA (Rupp.) L. Marsh Marigold 1. palustris L. ${ }^{47}$

13. GOPTIS Salisb. Goldthread

1. trifolia (L.) Salisb. ${ }^{34-47}$

17. AQUILEGIA (Tourn.) L. Columbine

1. canadensis L. ${ }^{47}$ (Wild C.)

18. DELPHINIUM (Tourn.) L. Larkspur

*1. Consolida L. (Field L.) B. V.

*2. ajacis $\mathrm{L}^{2-47}$ 
20. CIMICIFUGA L. Bugbane

2. racemosa (L.) Nutt. ${ }^{1}$ (Black Snakeroot, Black Cohosh.)

21. ACTAEA L. Baneberry. Cohosh

1. rubra (Ait.) Willd. ${ }^{47}$ (Red B.) A. spicata var. rubra Michx. ${ }^{1}$

2. alba (L.) Mill. ${ }^{47}$ (White B.)

FAMILY 62. MAGNOLIACEAE (Magnolia Family)

\section{LIRIODENDRON L. Tulip Tree}

1. Tulipifera L. ${ }^{1}$

FAMILY 66. BERBERIDACEAE (Barberry Family)

1. PODOPHYLLUM L. May Apple. Mandrake

1. peltatum $L .{ }^{1}$

4. CAULOPHYLLUM Michx. Blue Cohosh

1. thalictroides (L.) Michx. ${ }^{1}$ (Pappoose Root.)

5. BERBERIS (Tourn.) L. Barberry

*2. vulgaris L. ${ }^{1}$ (Common B.)

FAMILY 67. LAURACEAE (Laurel Family)

2. SASSAFRAS Nees.

1. variifolium (Salisb.) Ktze. S. officinale Nees. ${ }^{26}$

4. BENZOIN Fabric. Wild Allspice. Fever Bush

1. aestivale (L.) Nees. (Spice Bush, Benjamin Bush.) Lindera Benzoin Blume. ${ }^{26}$

ORDER XXVI PAPAVERALES

FAMILY 68. PAPAVERACEAE (Poppy Family)

1. SANGUINARIA (Dill.) L. Bloodroot

1. canadensis L. ${ }^{1}$

3. CHELIDONIUM (Tourn.) L. Celandine *1. majus L. ${ }^{1}$

4. GLAUCIUM (Tourn.) Hill. Horn Poppy. Sea Poppy

*1. flavum Crantz. ${ }^{1}$ G. luteum Scop.

5. PAPAVER (Tourn.) L. Poppy

*1. somniferum L. ${ }^{2}$ (Common P.)

*3. dubium L. ${ }^{2}$ 
FAMILY 69. FUMARIACEAE (Fumitory Family)

1. ADLUMIA Raf. Climbing Fumitory

1. fungosa (Ait.) Greene. A. cirrhosa Raf. ${ }^{1}$

2. DICENTRA Bernh.

1. Cucullaria (L.) Bernh. (Dutchman's Breeches.)

2. canadensis (Goldie.) Walp. (Squirrel Corn.)

3. CORYDALIS (Dill.) Medic.

1. sempervirens (L.) Pers. (Pale C.) C. glauca Pursh. ${ }^{1}$

4. FUMARIA (Tourn.) L. Fumitory

${ }^{*}$. officinalis L. ${ }^{1}$ (Common F.)

FAMILY 70. CRUCIFERAE (Mustard Family)

1. DRABA (Dill.) L.

*1. verna L. ${ }^{1}$ (Whitlow Grass.)

2. caroliniana Walt. ${ }^{1}$

2. BERTOERA D C.

*1. incana (L.) D C. Alyssum L. ${ }^{2}$

3. LOBULARIA Desv. Sweet Alyssum

*1. maritima (L.) Desv. Alyssum Lam. ${ }^{1}$

4. ALYSSUM (Tourn.) L.

*1. alyssoides L. A. calcynum $\mathrm{L}^{2}{ }^{2}$

6. THLASPI (Tourn.) L. Penny Cress

*1. arvense L. ${ }^{1}$ (Field or Mithridate Mustard.)

7. LEPIDIUM (Tourn.) L. Pepperwort. Peppergrass 1. virginicum L. ${ }^{52}$ (Wild Peppergrass.) L. virginianum L. ${ }^{1}$

*3. ruderale L. ${ }^{1}$

*5. campestre (L.) R. Br. ${ }^{1}$

*6. Draba L. ${ }^{1}$

8. CORONOPUS Ludwig. Wart Cress. Swine Cress *1. didymus (L.) Sm. Senebiera D C. didyma Pers. ${ }^{1}$

*2. procumbens Gilibert. Senebiera coronopus D C. ${ }^{1}$

10. CAPSELLA Medic. Shepherd's Purse

*1. Bursa-pastoris (L.) Medic. ${ }^{1}$

11. CAMELINA Crantz. False Flax

*1. sativa (L.) Crantz. ${ }^{1}$

*2. microcarpa Andrz. C. sylvestris Wallr. ${ }^{3}$ 
13. GAKILE (Tourn.) Ludwig. Sea Rocket

1. edentula (Bigel.) Hook. ${ }^{52}$ (American S.) C. americana Nutt. ${ }^{1}$

14. RAPHANUS (Tourn.) L. Radish

*1. Raphanistrum L. ${ }^{1-52}$ (Wild R., Jointed Charlock.)

*2. sativus L. ${ }^{1}$ (Radish.)

15. BRASSICA (Tourn.) L. Mustard. Turnip

*1. alba (L.) Boiss. ${ }^{1-52}$ (White M.)

*2. arvensis (L.) Ktze. (Charlock.) B. sinapistrum Boiss. ${ }^{1-52}$

*3. juncea (L.) Cosson. ${ }^{2}$

*5. nigra (L.) Koch. ${ }^{1-52}$ (Black M.)

*6. campestris L..$^{2-52}$ (Rutabaga.)

19. SISYMBRIUM (Tourn.) L. Hedge Mustard

*1. officinale (L.) Scop. ${ }^{1}$

*6. Sophia L. ${ }^{1}$

*7. Thalianum (L.) J. Gay. ${ }^{2}$ (Mouse-ear Cress.)

21. HESPERIS (Tourn.) L. Rocket

*1. matronalis L. (Dame's Violet.)

22. ERYSIMUM (Tourn.) L. Treacle Mustard

1. cheiranthoides L. ${ }^{?}$ (Worm-seed Mustard.)

23. RADICULA (Dill.) Hill. Water Cress

*1. Nasturtium-aquaticum (L.) Britten \& Rendle. (True W.) Nasturtium officinale R. Br. ${ }^{1}$

6. palustris (L.) Moench. Nasturtium palustre D C. ${ }^{1}$

*8. Armoracia (L.) Robinson. (Horse Radish.) Nasturtium R. Br. ${ }^{1}$

24. BARBAREA R. Br. Winter Cress

1. vulgaris $\mathrm{R} . \mathrm{Br}^{1}$ (Common W., Yellow Rocket.)

30. GARDAMINE (Tourn.) L. Bitter Cress

1. bulbosa (Schreb.) B S P. (Spring Cress.) C. rhomboidea D C. ${ }^{1}$

7. hirsuta L. ${ }^{1}$

8. parviflora L. C. hirsuta var. sylvatica Gray. ${ }^{1}$

9. pennsylvanica Muhl. C. flexuosa B. U.

31. ARABIS L. Rock Cress

3. glabra (L.) Bernh. (Tower Mustard.) Aperfoliata Lam. ${ }^{1}$ 
10. laevigata (Muhl.) Poir. ${ }^{1}$

11. canadensis L. ${ }^{1}$ (Sickle-pod.)

FAMILY 72. RESEDACEAE (Mignonette Family)

1. RESEDA (Tourn.) L. Mignonette. Dyer's Rocket

*1. Luteola L. ${ }^{46}$ (Dyer's Weed or Weld.)

*2. lutea L. ${ }^{37-46}$

ORDER XXVII SARRACENIALES

FAMILY 73. SARRACENIACEAE (Pitcher Plant Family) 1. SARRCENIA (Tourn.) L.

1. purpurea 20-34-46 (Side-saddle Flower, Pitcher Plant, Hun tsman's Cup.)

FAMILY 74. DROSERACEAE (Sundew Family)

1. DROSERA L. Sundew

1. rotundifolia L. ${ }^{34-46}$ (Round-leaved S.)

3. longifolia L. ${ }^{46}$ D. intermedia Hayne. ${ }^{34}$

ORDER XXVIII ROSALES

FAMILY 75. PODOSTEMACEAE (River Weed Family)

1. PODOSTEMUM Michx. River IVeed

1. ceratophyllum Michx. ${ }^{46}$

FAMILY 76. CRASSULACEAE (Orpine Family)

1. PENTHORUM (Gronov.) L. Ditch Stonecrop

1. sedoides L. ${ }^{46}$

3. SEDUM (Tourn.) L. Stonecrop. Orpine

*1. acre L. ${ }^{30-46}$ (Mossy S.)

*9. purpureum Tausch. ${ }^{46}$ (Garden O., Live-for-ever.) $S$. telephum L.'

FAMILY 77. SAXIFRAGACEAE (Saxifrage Family)

1. ASTIBBE Hamilton. False Goat's Beard

*1. A. japonica Gray. ${ }^{2}$ (Spiraea of Hort.)

4. SAXIFRAGA (Tourn.) L. Saxifrage

6. pennsylvanica L. ${ }^{45}$ (Swamp S.)

7. virginiensis Michx. ${ }^{45}$ (Early S.) 
8. GHR YSOSPLENIUM (Tourn.) L. Golden Saxifrage

1. americanum Schwein. ${ }^{45}$

9. PARNASSIA (Tourn.) L. Grass of Parnassus

3. caroliniana Michx. ${ }^{9-45}$

10. PHILADELPHUS L. Mock Orange or Syringa

* P. coronarius $\mathrm{L}^{3-45}$ (Syringa of Cult.)

14. RIBES L. Currant. Gooseberry

5. oxyacanthoides L. ${ }^{1}$ (Smooth G.)

oxyacanthoides var. calcicola Fernald. R. hirtellum

Michx. ${ }^{45}$

7. floridum L'Hér. ${ }^{1}$ (Wild Black C.) R. americanum Mill. ${ }^{45}$

13. triste Pall. (Swamp Red C.) R. rubrum var. subglandulosum Maxim. ${ }^{3}$

* R. odoratum Wendland. ${ }^{45}$ (Missouri C. of Cult.)

FAMILY 78. HAMAMELIDACEAE (Witch-hazel Family)

1. HAMAMELIS L. Witch-hazel

1. virginiana $L .{ }^{45}$

FAMILY 79. PLATANACEAE (Plane Tree Family)

1. PLATANUS (Tourn.) L. Sycamore. Buttonwood

1. occidentalis $\mathrm{L}^{45}$

FAMILY 80. ROSACEAE (Rose Family)

1. PHYSOCARPUS Maxim. Nine-bark

1. opulifolius (L.) Maixm. ${ }^{3}$

2. SPIRAEA (Tourn.) L.

6. latifolia Borkh. (Meadow-sweet.) S. salicifolia L. ${ }^{1}$

7. tomentosa L. ${ }^{1}$ (Hardhack, Steeple Bush.)

4. SORBARIA A. Br.

*1. sorbifolia (L.) A. Br. Spiraea L. ${ }^{30}$

6. PYRUS (Tourn.) L.

7. arbutifolia (L.) L. f. ${ }^{3}$

8. melanocarpa (Michx.) Willd. P. arbutifolia L. var. ${ }^{1}$

9. americana (Marsh.) D C. ${ }^{1}$ (American Mountain Ash.) 
7. AMELANCHIER Medic. Juneberry

1. canadensis (L.) Medic. (Shad Bush, Service Berry.) canadensis var. Botryapium (L. f.) T. \& G. ${ }^{1}$

2. oblongifolia (T. \& G.) Roem. A. canadensis var. T. \& G. ${ }^{1}$

3. spicata (Lam.) C. Koch. A. canadensis var. rotundifolia T. \& G. ${ }^{2}$

8. CRATAEGUS L. Hawthorn. White Thorn

*1. oxyacantha L.' (English H.)

2. Crus-galli L. ${ }^{1}$

8. punctata Jacq. C. tomentosa L. var. ${ }^{1}$

17. coccinea L. ${ }^{1}$

21. tomentosa L. C. tomentosa var. pyrifolia. ${ }^{1}$

10. FRAGARIA (Tourn.) L. Strawberry

1. virginiana Duchesne. ${ }^{1}$

2. vesca $L^{1-52}$

* F. grandiflora Ehrh. ${ }^{41}$ (Garden S.)

15. POTENTILLA L. Cinquefoil. Five-finger

2. monspeliensis $\mathrm{L}$. RW P

monspeliensis var. norvegica (L.) Rydb. $P$. norvegica L.'

7. $\operatorname{argentea}$ L. $^{1}$ (Silvery C.)

*10. recta $L$. ${ }^{1}$

15. fruticosa L. ${ }^{1}$ (Shrubby C.)

17. anserina L. ${ }^{1}$ (Silver Weed.)

19. canadensis L. ${ }^{1}$

canadensis var. simplex (Michx.) T. \& G.1

17. GEUM L. Avens

1. canadense Jacq. G. album Gmel. ${ }^{1}$

3. virginianum L. ${ }^{1}$

5. strictum Ait. ${ }^{1}$

8. rivale $\mathrm{L}^{1}$ (Water or Purple A.)

18. RUBUS (Tourn.) L. Bramble

1. idaeus L. var. aculeatissimus (C. A. Mey.) Regel \& Tiling. (Wild Red Raspberry.) R. strigosus Michx. ${ }^{1}$

3. occidentalis L. ${ }^{1-52}$ (Black R., Thimbleberry.)

4. odoratus L. ${ }^{1}$ (Purple Flowering R.)

7. triflorus Richards. ${ }^{1}$ (Dwarf R.)

9. allegheniensis Porter. $R$. villosus Ait. ${ }^{1}$ 
21. canadensis $\mathrm{L}^{1-52}$

34. hispidus $\mathrm{L},{ }^{1-52}$

37. villosus Ait. ${ }^{1-52}$ (Dewberry.) R. canadensis L. ${ }^{1}$

19. DALIBARDA Kalm.

1. repens L. Rubus Dalibarda. ${ }^{1}$

21. AGRIMONIA (Tourn.) L. Agrimony

2. striata Michx. A. Eupatoria L. ${ }^{1}$

3. mollis (T. \& G.) Britton. ${ }^{32}$

22. SANGUISORBA (Rupp.) L. Burnet

*3. minor Scop. (Garden B.) Poterium Sanguisorba L. ${ }^{2}$

23. ROSA (Tourn.) L. Rose

2. acicularis Lindl. Miss E. M. Round.

4. blanda Ait. ${ }^{7-37}$

*7. cinnamomea L. (Cinnamon R.) R W P

*8. canina L. ${ }^{3}$ (Dog R.)

*10. rubiginosa L. ${ }^{1}$ (Sweet Brier, Eglantine.)

* rubiginosa var. micrantha (Sm.) Lindl. R. micrantha Sm. ${ }^{1}$

13. carolina L. ${ }^{1}$

14. virginiana Mill. R. lucida Ehrh. ${ }^{1-52}$

15. humilis Marsh. ${ }^{3}$

24. PRUNUS (Tourn.) L. Plum Cherry

1. Serotina Ehrh. ${ }^{1}$ (Wild Black or Rum C.)

2. virginiana L. ${ }^{1}$ (Choke C.)

3. pennsylvanica L. f. ${ }^{1}$ (Wild Red, Bird, Fire or Pin C.)

*5. instititia L. (Bullace P.) P. spinosa L. ${ }^{1}$

6. maritima Wang. ${ }^{1-52}$ (Beach P.)

11. pumila L. ${ }^{1}$ (Sand C.)

16. americana Marsh. ${ }^{1}$ (Wild P.)

FAMILY 81. LEGUMINOSAE (Pulse Family)

6. GLEDITSIA L. Honey Locust

1. triacanthos L. ${ }^{1}$ (Honey Locust.)

7. CASSIA (Tourn.) L. Senna

1. marilandica L. ${ }^{8}$ (Wild S.)

5. Chamaecrista L. ${ }^{8-9}$ (Partridge Pea.)

7. nictitans L. ${ }^{8}$ (Wild Sensitive Plant.) 
9. BAPTISIA Vent. False Indigo

1. tinctoria (L.) R. Br. ${ }^{8-34}$ (Wild Indigo).

13. GROTOLARIA (Dill.) L. Rattle-box

1. sagittalis $\mathrm{L}^{8}$

17. LUPINUS (Tourn.) L. Lupine

1. perennis L. ${ }^{8}$ (Wild L.)

18. TRIFOLIUM (Tourn.) L. Clover. Trefoil

*1. arvense L. ${ }^{8}$ (Rabbit-foot or Stone C.)

*2. incarnatum L. ${ }^{3-8}$ (Crimson or Italian C.)

*3. pratense L. $^{8}$ (Red C.)

*4. medium L. ${ }^{8}$ (Zigzag C.)

6. reflexum L. (Buffalo C.)

8. repens L. ${ }^{8}$ (White C.)

*9. hybridum L. ${ }^{8-34}$ (Alsike C.)

*11. agrarium L. ${ }^{8}$ (Yellow or Hop C.)

*12. procumbens L. ${ }^{8}$ (Low Hop C.)

19. MELILOTUS (Tourn.) Hill. Melilot. Sweet Clover

*1. officinalis (L.) Lam. ${ }^{8}$ (Yellow M.)

*4. alba Desr. ${ }^{8}$ (White M.)

20. MEDICAGO (Tourn.) L. Medick

*1. sativa L. ${ }^{8}$ (Lucerne, Alfalfa.)

*3. Lupulina L. ${ }^{8}$ (Black M., Nonesuch.)

*4. arabica Huds. ${ }^{8}$ (Spotted M.) L. maculata Willd. ${ }^{1}$

*5. hispida Gaertn. ${ }^{8}$ (Bur Clover.) L. denticulata L. ${ }^{1}$

23. LOTUS (Tourn.) L. Bird's-foot Trefoil

L. tenuis Waldst. \& Kit. $^{31}$

28. TEPHROSIA Pers. Hoary Pea

1. virginiana (L.) Pers. ${ }^{8}$ (Goat's Rue, Catgut.)

30. ROBINIA L. Locust

1. Pseudo-Acacia L. ${ }^{8}$ (Common L., False Acacia.)

2. Viscosa Vent. (Clammy L.) Miss E. M. Round.

3. hispida L. ${ }^{8-14}$ (Bristly L., Rose Acacia.)

34. GLYCYRRHIZA (Tourn.) L. Liquorice

1. lepidota (Nutt.) Pursh. ${ }^{2}$ (Wild L.) 


\section{CORONILLA L.}

*1. varia $L .{ }^{3-8}$

38. DESMODIUM Desv. Tick Trefoil

1. nudiflorum (L.) D C. ${ }^{8}$

2. grandiflorum (Walt.) D C. D. acuminatum D C. ${ }^{8}$

4. rotundifolium (Michx.) D C. ${ }^{8}$

6. glabellum (Michx.) D C. D. humifusum Beck. ${ }^{8}$

7. canescens (L.) D C. ${ }^{8}$

8. bracteosum (Michx.) D C. D. cuspidatum T. \& G. ${ }^{8}$

12. Dillenii Darl. ${ }^{8}$

13. paniculatum (L.) D C. ${ }^{8-34}$

15. canadense (L.) D C..$^{8-34}$

16. sessilifolium (Torr.) T. \& G. ${ }^{8}$

17. rigidum (Ell.) D C. ${ }^{8-34}$

18. obtusum (Muhl.) D C. D. ciliare D C. ${ }^{8-34}$

19. marilandicum (L.) D C. ${ }^{8}$

39. LESPEDEZA Michx. Bush Clover

1. procumbens $\mathrm{Michx} .^{8}$

3. violacea (L.) Pers. ${ }^{8}$

5. Nuttallii Darl. ${ }^{8}$

7. Stuvei Nutt. ${ }^{8}$

8. virginica (L.) Britton. L. reticulata Pers. ${ }^{3-8}$

9. frutescens (L.) Britton. ${ }^{34}$ L. Stuvei var. intermedia Wats. ${ }^{8}$

11. hirta (L.) Hornem. L. polystachya Michx. ${ }^{8}$

12. capitata Michx. ${ }^{8-34}$

13. angustifolia (Pursh.) Ell. L. capitata var. ${ }^{1}$

42. VICIA (Tourn.) L. Vetch. Tare

*1. sativa L. ${ }^{8}$ (Spring V.)

*2. angustifolia (L.) Reichard..$^{2-8}$ (Common V.)

*4. tetrasperma (L.) Moench. ${ }^{8}$

*5. hirsuta (L.) S. F. Gray. ${ }^{2-8}$

7. Cracca L. ${ }^{8}$

43. LATHYRUS (Tourn.) L. Vetchling. Everlasting Pea

1. maritimus (L.) Bigel. ${ }^{8}$ (Beach Pea.)

2. palustris L. ${ }^{8}$ 
44. APIOS (Boerh.) Ludwig. Ground Nut. Wild Bean

1. tuberosa Moench. ${ }^{8}$

45. PHASEOLUS (Tourn.) L. Kidney Bean

1. polystachyus (L.) B S P. (Wild Bean.) P. diversifolia Pers. ${ }^{1}$

* P. vulgaris $\mathrm{L}^{2}{ }^{2}$

47. STROPHOSTYLES EII.

1. helvola (L.) Britton. S. angulosa Ell. ${ }^{8}$ Phaseolus diversifolia $\mathrm{L}^{\mathrm{t}}$

51. AMPHICARPA Ell. Hog Peanut

1. monoica (L.) Ell. ${ }^{8}$

2. Pitcheri T. \& G. ${ }^{32}$

\section{ORDER XXIX GERANIALES}

FAMILY 82. LINACEAE (Flax Family)

1. LINUM (Tourn.) L. Flax

*1. usitatissimum L. ${ }^{34}$ (Common F.)

6. sulcatum Riddell. ${ }^{1}$

7. striatum Walt. ${ }^{1-52}$

8. virginianum $L .{ }^{1}$

FAMILY 83. OXALIDACEAE (Wood Sorrel Family)

1. OXALIS L. Wood Sorrel

1. Acetosella L. ${ }^{1}$ (Common W.)

2. violacea L. ${ }^{1}$ (Violet W.)

5. stricta L. O. corniculata L. var. ${ }^{1}$

7. corniculata L. ${ }^{34}$ (Lady's Sorrel.)

FAMILY 84. GERANIACEAE (Geranium Family)

1. GERANIUM Cransebill

1. maculatum L. ${ }^{1}$ (Wild C.)

4. Robertianum L. ${ }^{1}$ (Herb Robert.)

6. carolinianum L. ${ }^{1}$

7. Bicknellii Britton.

2. ERODIUM L'Hér. Storksbill

*1. cicutarium (L.) L'Hér. ${ }^{1}$

TROPAEOLUM Nasturtium

* T. majus L. ${ }^{2}$ (Common N.) 
FAMILY 86. RUTACEAE (Rue Family)

1. ZANTHOXYLUM L. Prickly Ash

1. americanum Mill. ${ }^{1}$ (Northern P., Toothache-tree.)

FAMILY 87. SIMARUBACEAE (Quassia Family)

1. AILANTHUS Desf. Tree of Heaven

*1. glandulosa Desf. ${ }^{3}$

FAMILY 88. POLYGALACEAE (Milkwort Family)

1. POLYGALA (Tourn.) L. Milkwort

1. paucifolia Willd. $^{7}$ (Fringed Polygala, Flowering Wintergreen.)

2. polygama Walt. ${ }^{7-34}$

3. Senega L. ${ }^{7}$ (Seneca Snakeroot.)

5. sanguinea $L .{ }^{7}$

7. Nuttallii T. \& G. ${ }^{7-34}$

9. cruciata L. ${ }^{7}$

10. brevifolia Nutt. ${ }^{7}$

11. verticillata $L .{ }^{7}$

verticillata var. ambigua (Nutt.) Wood. ${ }^{7}$

FAMILY 89. EUPHORBIACEAE (Spurge Family)

6. ACALYPHA L. Three-seeded Mercury

1. virginica $L .{ }^{26}$

2. gracilens Gray. ${ }^{26}$ A. virginica var. Muell. Arg. ${ }^{1}$

12. EUPHORBIA L. Spurge

1. polygonifolia L. ${ }^{26}$ (Seaside S.)

8. Preslii Guss. ${ }^{26}$ E. hypericifolia L. ${ }^{1}$

9. hirsuta (Torr.) Wiegand. ${ }^{26}$

10. maculata L. ${ }^{26-34}$ (Milk Purslane.)

*26. Cyparissias L. ${ }^{26}$ (Cypress S.)

*28. Peplus L. ${ }^{26}$ (Petty S.)

FAMILY 90. CALLITRICHACEAE (WaterStarwort Family)

1. CALLITRICHE L. Water Starwort

2. palustris L. C. verna $\mathrm{L}^{1-52}$

3. heterophylla Pursh. ${ }^{1}$

ORDER XXX SAPINDALES

FAMILY 94. ANACARDIACEAE (Cashew Family)

1. RHUS L. Sumach

1. typhina L. ${ }^{7}$ (Staghorn S.) 
2. glabra L. ${ }^{7}$ (Smooth S.)

3. copallina L. ${ }^{7-34}$ (Dwarf S.)

4. Vernix L. (Poison S., or Dogwood.) R. venenata L. ${ }^{7}$

5. Toxicodendron L. ${ }^{7}$ (Poison Ivy, Poison Oak.) Toxicodendron var. radicans (L.) Torr. ${ }^{1}$

FAMILY 96. AQUIFOLIACEAE (Holly Family)

1. ILEX L. Holly

1. opaca Ait. ${ }^{1}$ (American H.)

6. verticillata (L.) Gray. ${ }^{1}$ (Black Alder, Winterberry.)

7. laevigata (Pursh.) Gray. ${ }^{1-52}$ (Smooth Winterberry.)

8. glabra (L.) Gray. ${ }^{1}$ (Inkberry.)

2. NEMOPANTHUS Raf. Mountain Holly

1. mucronata (L.) Trel. N. canadensis D C. ${ }^{1}$

FAMILY 97. CELASTRACEAE (Staff Tree Family)

1. EVONYMUS (Tourn.) L. Spindle Tree

1. atropurpureus Jacq. ${ }^{3}$ (Burning Bush, Wahoo.)

3. CELASTRUS L. Staff Tree. Shrubby Bitter-sweet

1. scandens L. ${ }^{1}$ (Waxwork, Climbing Bitter-sweet.)

FAMILY 98. STAPHYLEACEAE (Bladder Nut Family)

1. STAPHYLEA L. Bladder Nut

1. trifolia L. ${ }^{7}$ (American B.)

FAMILY 99. ACERACEAE (Maple Family)

1. ACER (Tourn.) L. Maple

1. pennsylvanicum L. ${ }^{7}$ (Striped M., Moosewood.)

2. spicatum Lam. ${ }^{7}$ (Mountain M.)

3. saccharum Marsh. ${ }^{i}$ (Sugaror Rock M.) A. saccharinum Wang. ${ }^{1}$

4. saccharinum L. ${ }^{7}$ (White or Silver M.) A. dasycarpum Ehrh. ${ }^{1}$

5. rubrum L. ${ }^{7}$ (Red or Swamp M.)

6. Negundo L. ${ }^{7}$ (Box Elder.) Negundo aceroides Moench.'

FAMILY 100. SAPINDAGEAE (Soapberry Family)

2. AESCULUS L. Horse-chestnut. Buckeye

*1. Hippocastanum L. (Common H.) 
FAMILY 101. BALSAMINACEAE (Touch-me-not Family) 1. IMPATIENS (Rivinius) L. Balsam. Jewelweed

1. pallida Nutt. ${ }^{1}$ (Pale Touch-me-not.)

2. biflora Walt. (Spotted Touch-me-not.) I. fulva Nutt. ${ }^{1}$

\section{ORDER XXXI RHAMNALES}

FAMILY 102. RHAMNAGEAE (Buckthorn Family)

2. RHAMNUS (Tourn.) L. Buckthorn

*2. cathartica L. ${ }^{1}$ (Common B.)

3. CEANOTHUS L. Red-root

1. americanus L. ${ }^{1}$ (New Jersey Tea.)

FAMILY 103. VITACEAE (Vine Family)

1. PSEDERA Neck. Virginia Creeper. Woodbine

1. quinquefolia (L.) Greene. Ampelopsis Michx. ${ }^{1}$

3. VITIS (Tourn.) L. Grape

1. labrusca L. ${ }^{1-52}$ (Northern Fox G.)

2. aestivalis Michx. ${ }^{-}$(Summer or Pigeon G.)

5. cordifolia Michx. ${ }^{1}$ (Frost or Chicken G.)

6. vulpina L. (River-bank or Frost G.) V. riparia Michx. ${ }^{1}$

ORDER XXXII MALVALES

FAMILY 104. TILIACEAE (Linden Family)

1. TILIA (Tourn.) L. Linden. Basswood

1. americana L. ${ }^{1}$ (Basswood.)

FAMILY 105. MALVACEAE (Mallow Family)

1. ABUTILON (Tourn.) Mill. Indian Mallow

*1. Theophrasti Medic. (Velvet Leaf.) A. Avicennae Gaertn. ${ }^{1-52}$

6. ALTHEA L. Marsh Mallow

*1. officinalis L. (Marsh Mallow.)

7. MALVA (Tourn.) L. Mallow

*1. rotundifolia L. ${ }^{1-52}$ (Common M., Cheeses.)

*2. verticillata $\mathrm{L}$. M. crispa $\mathrm{L} .{ }^{1}$

*3. sylvestris L. ${ }^{1}$ (High M.)

*4. moschata L.' (Musk M.) 


\section{HIBISCUS L. Rose Mallow}

*1. syriacus L. ${ }^{1}$ (Shrubby Althea of gardens.)

2. Moscheutos L. ${ }^{1}$ (Swamp R.)

*7. Trionum L. ${ }^{1}$ (Flower-of-an-hour.)

\section{ORDER XXXIII VIOLALES}

FAMILY 107. HYPERICACEAE (St. John's-wort Family) 2. HYPERICUM (Tourn.) L. St. John's-wort

*3. perforatum L. ${ }^{7}$ (Common S.)

4. punctatum Lam. H. corymbosum Muhl. ${ }^{1} H$. maculatum Walt. ${ }^{7-34}$

10. adpressum Bart. ${ }^{7}$

14. ellipticum Hook. ${ }^{7}$

16. boreale (Britton) Bicknell. ${ }^{7-34}$

17. mutilum L. ${ }^{7}$

19. majus (Gray.)Britton. H. canadense var. Gray. ${ }^{7}$

20. canadense L. ${ }^{7-34}$

22. gentianoides (L.) B S P. ${ }^{34}$ (Orange Grass, Fineweed.) H. sarothra Michx. ${ }^{1}$ H. nudicaule Walt. ${ }^{7}$

23. virginicum L. ${ }^{7-34}$ (Marsh S.) Elodes campanulata Pursh. ${ }^{1}$

FAMILY 108. ELATINACEAE (Waterwort Family)

1. ELATINE L. Waterwort

1. americana (Pursh.) Arn. ${ }^{7}$

FAMILY 109. CISTACEAE (Rockrose Family)

1. HELIANTHEMUM (Tourn.) Mill. Rockrose

1. canadense L.?) Michx. ${ }^{7}$ (Frostweed.)

2. majus B S P. ${ }^{7}$ (Frostweed.)

2. HUDSONIA L.

1. ericoides L. ${ }^{7}$

2. tomentosa Nutt. ${ }^{7}$

3. LECHEA (Kalm.) L. Pinweed

1. villosa E. U. L. major Michx. ${ }^{\top}$

2. minor L. ${ }^{3-7}$ L. Novae-Caesareae Austin. ${ }^{1}$

3. in termedia Leggett. ${ }^{7}$

5. maritima Leggett. ${ }^{7}$ L. thymifolia Pursh. ${ }^{1}$

6. tenuifolia Michx. ${ }^{7}$ L. minor Lam. var. ${ }^{1}$ 
FAMILY 110. VIOLACEAE (Violet Family)

2. VIOLA (Tourn.) L. Violet. Heart's-ease

1. pedata L. ${ }^{34}$ (Bird-foot V.) V. pedata var. bicolor Pursh. ${ }^{1}$ pedata var. lineariloba D C. $\quad$. pedata $\mathrm{L}^{1}$

2. cucullata Ait. V. palmata var. Gray. ${ }^{1}$

9. palmata $L .{ }^{1}$

15. fimbriatula Sm. V. ovata Nutt. $^{3}$

16. sagittata Ait. ${ }^{1}$

24. lanceolata L. ${ }^{1-52}$ (Lance-leaved V.)

25. primulifolia L. ${ }^{1-52}$ (Primrose-leaved V.)

27. blanda Willd. ${ }^{1}$

30. rotundifolia Michx. ${ }^{1}$ (Round-leaved or Early Yellow V.)

*31. odorata L. ${ }^{1}$ (English or Sweet V.)

34. pubescens Ait. ${ }^{1}$ (Downy Yellow V.)

35. scabriuscula Schwein (Smooth Yellow V.) V. pubescens Ait. var. ${ }^{1}$

37. striata Ait. ${ }^{1}$

38. conspersa Reichenb. V. canina var. Muhlenbergii Gray. ${ }^{1}$

40. arenaria D C.

*43. tricolor L. (Pansy, Heart's-ease.)

ORDER XXXIV OPUNTIALES

FAMILY 113. CACTACEAE (Cactus Family)

2. OPUNTIA (Tourn.) Mill. Prickly Pear. Indian Fig

1. vulgaris Mill. ${ }^{1}$

ORDER XXXV MYRTALES

FAMILY 114. THYMELAEACEAE (Mezereum Family)

1. DIRCA L. Leatherwood. Moosewood

1. palustris L. ${ }^{26}$ (Wicopy.)

2. DAPHNE L. Mezereum

*1. mezereum L. ${ }^{26}$

FAMILY 116. LYTHRACEAE (Loosestrife Family)

2. ROTALA L.

1. ramosior (L.) Koehne. Ammannia humilis Michx. ${ }^{1}$ 
4. DECODON J. F. Gmel. Swamp Loosestrife

1. verticillatus (L.) Ell. ${ }^{52}$ (Water Willow.) Nesaea, Commerson, verticillata $\mathrm{H}$. B. K. ${ }^{1}$

5. LYTHRUM L. Loosestrife

1. Hyssopifolia L. ${ }^{1}$

3. alatum Pursh. ${ }^{1}$

*4. Salicaria L. ${ }^{1}$ (Spiked L.)

6. CUPHEA P. Br.

1. petiolata (L.) Koehne. (Clammy C.) C. viscosissima Jacq. ${ }^{1}$

FAMILY 117. MELASTOMACEAE (Melastoma Family)

1. RHEXIA L. Deer Grass. Meadow Beauty

1. virginica $\mathrm{L} .{ }^{34}$

FAMILY 118. ONAGRACEAE (Evening Primrose Family)

2. LUDVIGIA L. False Loosestrife

1. alternifolia L. ${ }^{1}$ (Seedbox.)

8. palustris (L.) Ell.1-52 (Water Purslane.)

3. EPILOBIUM L. Willow-herb

1. angustifolium L. (Great W., Fireweed.) E. spicatum Lam. ${ }^{1}$ Chamaenerium Scop. ${ }^{34}$

*2. hirsutum L. ${ }^{1}$

3. molle Torr. ${ }^{11}$

4. densum Raf. E. palustre L. var. lineare. ${ }^{1}$

5. palustre L.

6. coloratum Muhl. ${ }^{1}$

7. adenocaulon Haussk.

4. OENOTHERA L. Evening Primrose

5. biennis L. ${ }^{34}$ (Common E.)

11. pumila L. ${ }^{1}$ Kneiffia Spach. ${ }^{34}$

13. fruticosa L. ${ }^{1}$ (Sundrops.)

8. CIRCAEA (Tourn.) L. Enchanter's Nightshade

1. lutetiana L. ${ }^{1}$

3. alpina L. ${ }^{1}$

FAMILY 119. HALORAGIDAGEAE (Water Milfoil Family)

1. MYRIOPHYLLUM (Vaill.) L. Water Milfoil

2. spicatum L. ${ }^{1}$ 
6. scabratum Michx. ${ }^{1} \quad M$. pinnatum (Walt.) B S P.

8. humile (Raf.) Morong. M. abmiguum var. limosum Nutt. ${ }^{1}$

humile forma natans (D C.) Fernald. M. ambiguum Nutt. ${ }^{1}$

humile forma capillaceum (Torr.) Fernald. M. ambiguum var. capillaceum T. \& G. ${ }^{1}$

9. tenellum Bigel. ${ }^{1}$

2. PROSERPINAGA L. Mermaid Weed

1. palustris $L \cdot{ }^{1-52}$

2. pectinata Lam. ${ }^{1}$

ORDER XXXVI UMBELLALES

FAMILY 120. ARALIACEAE (Ginseng Family)

1. ARALIA (Tourn.) L.

2. racemosa L. ${ }^{1}$ (Spikenard.)

3. hispida Vent. ${ }^{34}$ (Bristly Sarsaparilla, Wild Elder.)

4. nudicaulis L. ${ }^{1}$ (Wild Sarsaparilla.)

2. PANAX L. Ginseng

1. quinquefolium L. (Ginseng.) Aralia Tourn. ${ }^{1}$

2. trifolium L. (Dwarf G., Ground-nut.) Aralia Tourn. ${ }^{1}$

FAMILY 121. UMBELLIFERAE (Parsley Family)

2. SANICULA (Tourn. L.) Sanicle. Black Snakeroot

1. marilandica L. ${ }^{5}$

2. gregaria Bicknell.

3. canadensis L. ${ }^{1}$

3. HYDROCOTYLE (Tourn.) L. Water Pennywort 1. umbellata L. ${ }^{5}$

3. verticillata Thunb. H. interrupta Muhl. ${ }^{1}$

5. americana L. ${ }^{5}$

7. OSMORHIZA Raf. Sweet Cicely

1. Claytoni (Michx.) Clarke. O. brevistylis D C. ${ }^{5}$

2. longistylis (Torr.) D C. ${ }^{5}$

9. CONIUM L. Poison Hemlock

*1. maculatum L. ${ }^{5}$ 
10. PTILIMNIUM Raf. Mock Bishop's-weed

1. capillaceum (Michx.) Raf. Discopleura D C. ${ }^{5}$

11. AEGOPODIUM L. Goutweed

*1. Podagraria L. ${ }^{5}$

12. CICUTA L. Water Hemlock

1. maculata L. ${ }^{5-34}$ (Spotted Cowbane, Musquash Root, Beaver Poison.)

3. bulbifera L. ${ }^{5-34}$

13. GARUM L. Caraway

*1. Carvi L. ${ }^{2-5}$ (Caraway.)

16. SIUM (Tourn.) L. Water Parsnip

1. cicutaefolium Schrank. ${ }^{5-34}$

cicutaefolium var. Carsonii Durand. S. Carsonii Durand. ${ }^{5}$

17. GR YPTOTAENIA D C. Honewort

1. canadensis (L.) D C. ${ }^{5}$

18. ZIZIA Koch.

1. aurea (L.) Koch. ${ }^{5}$ (Golden Alexanders.)

3. cordata (Walt.) D C. ${ }^{5}$

19. FOENIGULUM (Tourn.) Hill. Fennel

*1. vulgare Hill. ${ }^{2}$

21. TAENIDIA Drude.

1. integerrima (L.) Drude. Pimpinella Benth. ${ }^{5}$

24. SCANDIX (Tourn.) L. Venus' Comb

*1. Pecten-Veneris L. ${ }^{2}$

26. LILAEOPSIS Greene.

1. lineata (Michx.) Greene. Crantzia Nutt. ${ }^{5}$

30. AETHUSA L. Fool's Parsley

*1. Cynapium L. ${ }^{5}$

31. COELOPLEURUM Ledeb.

1. actaeifolium (Michx.) Coult. \& Rose. C.Gmelini Ledeb. ${ }^{5}$

37. PASTINAGA L. Parsnip

*1. sativa L. ${ }^{5}$ 
38. LEVISTICUM (Rivinius) Hill. Lovage

L. scothicum L. ${ }^{5}$ Ligusticum L. ${ }^{1}$

40. HERACLEUM L. Cow Parsnip

1. lanatum Michx. ${ }^{5}$

44. ANGELICA L. Angelica

4. atropurpurea L. ${ }^{5}$

46. DAUCUS (Tourn.) L. Carrot

*1. Carota L. ${ }^{5}$

BIFORA Hoffm.

B. radians Bieb. ${ }^{5}$

FAMILY 122. CORNACEAE (Dogwood Family)

1. CORNUS (Tourn.) L. Cornel. Dogwood

1. canadensis L. ${ }^{24}$ (Dwarf C., Bunchberry)

3. florida L. ${ }^{24}$ (Flowering D.)

4. circinata L'Hér. ${ }^{24}$ (Round-leaved C. or D.)

5. Amomum Mill. ${ }^{24-34}$ (Silky C., Kinnikinnick.) C. sericea

L. ${ }^{1}$

Amomum forma purpusi Koehne. ${ }^{24}$

8. stolonifera Michx. ${ }^{1}$ (Red-osier D.)

10. paniculata L'Hér. ${ }^{24}$

11. alternifolia L. f. ${ }^{24}$

2. NYSSA L. Tupelo. Pepperidge. Sour Gum

1. sylvatica Marsh. ${ }^{24}$ (Black Gum.) N. multiflora Wang. ${ }^{1}$ sylvatica var. biflora Sarg. Miss E. M. Round.

Subclass II Metachlamydeae

ORDER XXXVII ERICALES

FAMILY 123. ERICACEAE (Heath Family)

1. CLETHRA (Gronov.) L. White Alder

1. alnifolia L. ${ }^{4-34}$ (Sweet Pepperbush.)

2. CHIMAPHILA Pursh. Pipsissewa

1. umbellata (L.) Nutt. ${ }^{4-34}$ (Prince's Pine, Pipsissewa.)

2. maculata (L.) Pursh. ${ }^{4-34}$ (Spotted Wintergreen.)

3. MONESES Salisb. One-flowered Pyrola

1. uniflora (L.) Gray. ${ }^{1}$ M. grandiflora Salisb. ${ }^{4}$ 
4. PYROLA (Tourn.) L. Wintergreen. Shin Leaf

2. secunda L. ${ }^{4}$

3. chlorantha Sw. ${ }^{4}$

4. elliptica Nutt. ${ }^{4}$ (Shin Leaf.)

5. americana Sweet. P. rotundifolia L. ${ }^{4}$

5. MONOTROPA Indian Pipe. Pinesap

1. uniflora.L. ${ }^{4}$ (Indian Pipe, Corpse Plant.)

2. Hypopitys L. ${ }^{4}$ (Pinesap, False Beech Drops.)

\section{RHODODENDRON L.}

2. viscosum (L.) Torr. ${ }^{4-9}$ (Clammy Azalea, White Swamp Honeysuckle.)

viscosum var. glancum (Michx.) Gray. ${ }^{4}$

3. nudifiorum (L.) Torr. ${ }^{4-34}$ (Purple A., Pinxter Flower.)

6. canadense (L.) B S P. (Rhodora.) R. Rhodora L. ${ }^{4-9}$

7. maximum L. ${ }^{4-34}$ (Great Laurel.)

13. KALMIA L. Laurel (of America)

1. Iatifolia L.4-34 (Mountain L., Calico Bush, Spoon-wood.)

2. angustifolia L. ${ }^{4}$ (Sheep L., Lambkill.)

4. polifolia Wang. (Pale L.) K. glauca Ait. ${ }^{27}$

16. LEUCOTHOË D. Don. Fetter Bush

4. racemosa (L.) Gray. ${ }^{4}$

17. ANDROMEDA L.

2. glaucophylla Link. ${ }^{27}$ (Bog Rosemary.)

18. LYONIA Nutt.

2. mariana (L.) D. Don. (Stagger Bush.) Andromeda L. ${ }^{4}$

3. ligustrina (L.) D C. (Male Berry.) Andromeda Muhl. ${ }^{4-52}$

19. CHAMAEDAPHNE Moench. Leather Leaf. Cassandra

1. calyculata (L.) Moench. Cassandra D. Don. ${ }^{4}$

21. EPIGAEA L. Ground Laurel. Trailing Arbutus 1. repens $L^{4-9}$ (Mayflower.)

22. GAUlTHERIA (Kalm.) L. Aromatic Wintergreen 1. procumbens L. ${ }^{4-38}$ (Teaberry, Checkerberry.)

23. ARGTOSTAPHYLOS Adans. Bearberry

1. Uva-ursi (L.) Spreng. ${ }^{4}$ (Bearberry.) 
24. Calluna Salisb. Heather. Ling *1. vulgaris (L.) Hull. ${ }^{3-4}$

25. CHIOGENES Salisb. Creeping Snowberry

1. hispidula (L.) T. \& G. ${ }^{1}$ (Moxie Plum, Capillaire.) C. serpyllifolia Salisb. ${ }^{4}$

26. GAYLUSSACIA H B K. Huckleberry

2. dumosa (Andr.) T. \& G. ${ }^{4-34}$ (Dwarf H.) dumosa var. hirtella (Ait. f.) Gray. ${ }^{4-52}$

3. frondosa (L.) T. \& G. ${ }^{4}$ (Dangleberry.)

5. baccata (Wang.) C. Koch. (Black H.) G. resinosa T. \& G. ${ }^{4}$

27. VACGINIUM L. Blueberry. Cranberry

7. pennsylvanicum Lam. ${ }^{4}$ (Low Sweet B., Early Sweet B.)

9. vacillans Kalm. ${ }^{4}$ (Late Low B.)

10. corymbosum L. ${ }^{4}$ (High or Swamp B.) corymbosum var. amoenum (Ait.) Gray. ${ }^{4}$ corymbosum var. pallidum (Ait.) Gray. ${ }^{4-34}$

11. atrococcum (Gray.) Heller. ${ }^{34}$ (Black High B.) V.corymbosum var. Gray. ${ }^{4}$

18. oxycoccos L. ${ }^{4}$ (Small Cranberry.) Oxycoccos palustris Pers. ${ }^{30}$

19. macrocarpon Ait. ${ }^{4}$ (Large or American Cranberry.)
ORDER XXXVIII PRIMULALES

FAMILY 125. PLUMBAGINACEAE (Leadwort Family)

1. LIMONIUM (Tourn.) Hill. Sea Lavender. Marsh Rosemary

1. carolinianum (Walt.) Britton. Statice Limonium var. Gray. ${ }^{15}$

FAMILY 126. PRIMULACEAE (Primrose Family)

3. HOTTONIA (Boerh.) L. Featherfoil. Water Violet 1. inflata Ell. ${ }^{15}$

4. SAMOLUS (Tourn.) L. Water Pimpernel. Brookweed

2. floribundus H B K. ${ }^{52}$ S. Valerandi var. americanus Gray..$^{\circ}$

5. LYSIMACHIA (Tourn.) L. Loosestrife *1. vulgaris $L^{15}$ 
3. quadrifolia L. ${ }^{15-34}$

x producta (Gray.) Fernald. ${ }^{41}$ L. foliosa Small.

4. terrestris (L.) B S P. ${ }^{52}$ L. stricta Ait. ${ }^{15}$

*5. Nummularia L. ${ }^{15}$ (Moneywort.)

6. thyrsi:aora L.15 (Tufted L.)

6. STEIRONEMA Raf.

1. ciliatum (L.) Raf. ${ }^{15}$

4. lanceolatum (Walt.) Gray. ${ }^{15}$ lanceolatum var. hybridum (Michx.) Gray. ${ }^{1}$

7. TRIENTALIS L. Chickweed Wintergreen

1. americana (Pers.) Pursh. ${ }^{15}$ (Star Flower.)

9. ANAGALLIS (Tourn.) L. Pimpernel *1. arvensis L. ${ }^{15}$ (Common P.)

ORDER XXXIX EBENALES

FAMILY 128. EBENACEAE (Ebony Family)

1. DIOSPYROS L. Persimmon

1. virginiana L. ${ }^{1}$ (Common P.)

\section{ORDER XL GENTIANALES}

FAMILY 130. OLEACEAE (Olive Family)

1. FRAXINUS (Tourn.) L. Ash

1. americana L. ${ }^{15}$ (White A.)

3. pennsylvanica Marsh. ${ }^{15}$ (Red A.) F. pubescens Lam. pennsylvanica var. lanceolata (Borkh.) Sarg. ${ }^{15}$ (Green

A.) F. viridis Michx. f.

7. nigra Marsh. (Black A.) F. sambucifolia Lam. ${ }^{15}$

2. SYRINGA L. Lilac

*1. vulgaris $\mathrm{L}^{15}$ (Common L.)

5. LIGUSTRUM (Tourn.) L. Privet

*1. vulgare L. ${ }^{15}$ (Privet or Prim.)

FAMILY 132. GENTIANACEAE (Gentian Family)

1. SABATIA Adans.

7. stellaris Pursh. ${ }^{1}$

9. dodecandra (L.) B S P. S. chloroides Pursh..$^{34}$ 
3. GENTIANA (Tourn.) L. Gentian

1. crinita Froel. ${ }^{1}$ (Fringed G.)

8. Andrewsii Griseb. ${ }^{1}$ (Closed G.)

7. BARTONIA Muhl.

2. virginica (L.) B S P. ${ }^{34}$ B. tenella Muhl. ${ }^{1}$

9. MENYANTHES (Tourn.) L. Buckbean

1. trifoliata L. ${ }^{1-52}$

10. NYMPHOIDES (Tourn.) Hill. Floating Heart

1. lacunosum (Vent.) Fernald. Limnanthemum Griseb.1-52 FAMILY 133. APOCYNACEAE (Dogbane Family)

2. VINCA L. Periwinkle

*1. minor L. ${ }^{30}$ (Common P., Myrtle.)

4. APOCYNUM (Tourn.) L. Dogbane. Indian Hemp

1. androsaemifolium L. ${ }^{15}$ (Spreading D.)

3. cannabinum L. ${ }^{15}$ (Indian Hemp.) cannabinum var. pubescens (R. Br.) D C. ${ }^{41}$

FAMILY 134. ASCLEPIADACEAE (Milkweed Family)

2. ASCLEPIAS (Tourn.) L. Milkweed. Silkweed

1. tuberosa L. ${ }^{15}$ (Butterfly Weed, Pleurisy-root.)

4. purpurascens L. ${ }^{15}$ (Purple M.)

5. incarnata L. (Swamp M.) incarnata var. pulchra (Ehrh.) Pers. ${ }^{15-34}$

7. syriaca L. ${ }^{15}$ (Common M. or Silkweed.) A. cornuti Dene. ${ }^{1}$

9. amplexicaulis Sm. A. obtusifolia Michx. ${ }^{15}$

11. phytolaccoides Pursh. ${ }^{15}$ (Poke M.)

14. quadrifolia Jacq. ${ }^{15}$

16. verticillata $L .{ }^{15}$

5. GYNANCHUM L.

*1. nigrum (L.) Pers. Vincetoxicum Moench..$^{15}$

ORDER XLI POLEMONIALES

FAMILY 135. CONVOLVULACEAE (Ccnvolvulus Family)

4. IPOMEA L. Morning Glory

*2. coccinea $\mathrm{L} .{ }^{2}$

*4. purpurea (L.) Roth. ${ }^{2}$ (Common M.) 
5. CONvOlvUluS (Tourn.) L. Bindweed

1. spithamaeus L. C. spithcmaea Pursh. ${ }^{1}$

3. sepium L..$^{1-52}$ (Hedge B.)

*4. arvensis L. ${ }^{1}$ (Field B.)

6. CUSGUTA (Tourn.) L. Dodder. Love Vine

4. obtusiflora H B K. C. chlorocarpa Engelm. ${ }^{1}$

8. Coryli Engelm. C. inflexa Engelm. ${ }^{1}$

9. Gronovii Willd. ${ }^{1}$

12. compacta Juss. ${ }^{1}$

FAMILY 136. POLEMONIACEAE (Polemonium Family)

1. PHLOX L.

1. paniculata $\mathrm{L}^{3-28}$

FAMLY 137. HYDROPHYLLACEAE (Waterleaf Family)

4. PHACELIA Juss.

2. Purshii Buckley. 28

FAMILY 138. BORAGINACEAE (Borage Family)

2. GYNOGLOSSUM (Tourn.) L. Hound's Tonzue

*1. officinale L. ${ }^{14}$ (Common H.)

3. LAPPULA (Rivinius) Moench. Stickseed

1. virginiana (L.) Greene. (Beggar's Lice.) Echinospermum virginicum Lehm. ${ }^{14}$

6. SYMPHYTUM (Tourn.) L. Comfrey

*1. officinale L. ${ }^{14}$ (Common C.)

7. LYCOPSIS L. Bugloss

*1. arvensis L. ${ }^{14}$ (Small B.)

8. MYSOTIS (Rupp.) L. Scorpion Grass. Forget-menot

*1. scorpicides L. (True F.) M. palustris With. ${ }^{14}$

2. laxa Lehm.14

3. $\operatorname{arvensis~(L.)~Hill.~}{ }^{14}$

6. virginica (L.) B S P. M. verna Nutt.14

10. LITHOSPERMUM (Tourn.) L. Gromwell. Puccoon

*1. arvense L. ${ }^{1 *}$ (Corn G.) 
*2. officinale L. ${ }^{14}$ (Common G.)

5. canescens (Michx.) Lehm. (Puccoon.)

11. ONOSMODIUM Michx. False Gromwell

1. virginianum (L.) A. D C. ${ }^{14}$

12. ECHIUM (Tourn.) L. Viper's Bugloss

*1. vulgare L. ${ }^{14}$ (Blue-weed, Blue Devil.)

FAMILY 139. VERBENACEAE (Vervain Family)

1. VERBENA (Tourn.) L. Vervain

*1. officinalis L. ${ }^{1}$ (European V.)

2. urticaefolia L. ${ }^{29}$ (White V.)

4. hastata L. ${ }^{29}$ (Blue V.)

FAMILY 140. LABIATAE (Mint Family)

1. AJUGA L. Bugle Weed

*2. genevensis L.

2. TEUCRIUM (Tourn.) L. Germander

1. canadense L. ${ }^{1}$ (American G., Wood Sage.)

4. TRICHOSTEMA L. Blue Curls

1. dichotomum L. ${ }^{1}$ (Bastard Pennyroyal.)

2. lineare Walt. ${ }^{1}$

5. SGUTELLARIA L. Skullcap

1. lateriflora L. ${ }^{34}$ (Mad-dog S.)

8. galericulata L. ${ }^{1}$

9. integrifolia L. ${ }^{1}$

6. MARRUBIUM (Tourn.) L. Horehound

*1. vulgare L. ${ }^{1}$ (Common H.)

9. NEPETA L. Cat Mint

*1. Cataria L. ${ }^{1}$ (Catnip.)

*2. hederacea (L.) Trevisan. (Ground Ivy, Gill-over-theGround.) N. Glechoma Benth. ${ }^{1}$

10. DRACOCEPHALUM (Tourn.) L. Dragon Head

1. parviflorum Nutt. ${ }^{51}$

11. PRUNELLA L. Self-heal

1. vulgaris L. ${ }^{34}$ (Heal-all, Carpenter-weed.) 
12. PHYSOSTEGIA Benth. False Dragon Hearl

1. virginiana (L.) Benth. ${ }^{1}$

\section{GALEOPSIS L. Hemp Nettle}

*1. Tetrahit L. ${ }^{1}$ (Common H.)

*2. Ladanum L. ${ }^{1}$ (Red H.)

16. LAMIUM L. Dead Nettle

*1. amplexicaule L. ${ }^{2}$ (Henbit.)

*2. purpureum L. ${ }^{1}$

*3. album L. ${ }^{1}$

17. LEONURUS L. Motherwort

*1. Cardiaca L. ${ }^{1}$ (Common M.)

18. BALLOTA L. Fetid Horehound

*1. nigra L. ${ }^{1}$ (Black Horehound.)

19. STACHYS (Tourn.) L. Hedge Nettle

*1. annua L. ${ }^{2}$

3. hyssopifolia Michx. ${ }^{1}$

5. tenuifolia Willd.

tenuifolia var. aspera (Michx.) Fernald. S. aspera Michx. ${ }^{1}$

9. palustris L. ${ }^{1}$ (Woundwort.)

10. cordata Riddell. ${ }^{1}$

S. italica Mill.2.

20. SALVIA (Tourn.) L. Sage

2. azurea Lam. var. grandiflora Benth. Miss E. M. Round.

21. MONARDA L. Horse Mint

1. didyma L. (Oswego Tea, Bee Balm.) Miss E. M. Round

4. mollis L. M. fistulosa var. Benth. ${ }^{1}$

23. HEDEOMA Pers. Mock Pennyroyal

1. pulegioides (L.) Pers. ${ }^{1}$ (American Pennyroyal.)

24. MELISSA (Tourn.) L. Balm

*1. officinalis L. (Common B.)

25. SATUREJA (Tourn.) L. Savory. Calamint

6. vulgaris (L.) Fritsch. (Basil.) Calamintha Clinopodium Benth. ${ }^{1}$ 
28. PYCNANTHEMUM Michx. Mountain Mint. Basil.

5. flexuosum (Walt.) B S P. P. linifolinm Pursh. ${ }^{1}$

6. virginianum (L.) Durand \& Jackson. P. lanceolatum Pursh. ${ }^{1}$ Koellia Mac M..$^{30}$

10. incanum (L.) Michx. ${ }^{1}$

12. muticum (Michx.) Pers. ${ }^{1}$

29. THYMUS (Tourn.) L. Thyme

*1. Serpyllum L. ${ }^{1}$ (Creeping T.)

31. LYCOPUS (Tourn.) L. Water Horehound

1. virginicus L. ${ }^{34}$ (Bugle Weed.)

5. lucidus Turoz. var. americanus Gray. L. lucidus L. ${ }^{1}$

7. americanus Muhl. ${ }^{34}$ L. sinuatus Ell. ${ }^{1}$

32. MENTHA (Tourn.) L. Mint

*4. spicata L. (Spearmint.) M. viridis L. ${ }^{1}$

*5. piperita L. ${ }^{1}$ (Peppermint.)

*9. Cardiaca Gerarde.

11. arvensis L. ${ }^{2-34}$

arvensis var. canadensis (L.) Briquet. M. canadensis L. ${ }^{1}$

33. COLLINSONIA L. Horse Balm

1. canadensis L. ${ }^{1}$ (Rich-weed, Stone-root.)

FAMILY 141. SOLANACEAE (Nightshade Family)

1. SOLANUM (Tourn.) L. Nightshade

1. Dulcamara L. ${ }^{1}$ (Bittersweet.)

3. nigrum L. ${ }^{1}$ (Common N.)

*7. rostratum Dunal. ${ }^{1}$ (Buffalo Bur.)

3. PHYSALIS L. Ground Cherry

3. angulata L. ${ }^{1}$

4. pubescens $\mathrm{L}^{1}{ }^{1}$

8. viscosa L. ${ }^{1}$

*10. Alkekengi L. ${ }^{1}$ (Winter Cherry.)

11. heterophylla Nees.

13. subglabrata Mackenzie \& Bush. P. philadelphica. ${ }^{2}$

14. virginiana Mill. ${ }^{1}$

4. NICANDRA Adans. Apple of Peru *1. physalodes (L.) Pers. ${ }^{1-52}$ 
5. LYCIUM L. Matrimony Vine

*1. halimifolium Mill. (Common M.) L. vulgare Dunal. ${ }^{31}$

6. HYOSCYAMUS (Tourn.) L. Henbane

*1. niger L. ${ }^{1}$ (Black H.)

7. DATURA L. Jamestown or Jimson Weed. Thorn Apple

*1. Stramonium L. ${ }^{1}$ (Stramonium.)

*2. Tatula L. ${ }^{1-52}$ (Purple T.)

*3. Metel L. ${ }^{2}$

FAMILY 142. SCROPHULARIACEAE (Figwort Family)

1. VERBASCUM (Tourn.) L. Mullein

*1. Thapsus L. ${ }^{29}$ (Common M.)

*3. Blattaria L. ${ }^{29}$ (Moth M.)

*5. Lychnitis L. ${ }^{29}$ (White M.)

2. LINARIA (Tourn.) Hill. Toadflax

*1. vulgaris Hill. ${ }^{29}$ (Ramsted, Butter and Eggs.)

3. canadensis (L.) Dumont. ${ }^{29}$

*5. Elatine (L.) Mill. ${ }^{29}$

5. SGROPHULARIA (Tourn.) L.. Figwort

1. marilancieica L. ${ }^{29}$ S. nodosa L. ${ }^{1}$

2. leporella Bicknell. ${ }^{29-41}$

6. PENTSTEMON (Mitchell) Ait. Beard-tongue

1. hirsutus (L.) Willd. P. pubescens Solander. ${ }^{29}$

7. laevigatus Ait. Viiss L. Hixon laevigatus var. Digitalis (Sweet) Gray. ${ }^{29}$

7. CHELONE (Tourn.) L. Turtlehead. Snakehead

1. Slabra L. ${ }^{29}$ (Balmony.)

9. MIMULUS L. Monkey Flower

1. ringens $\mathrm{L}^{29}$

2. alatus Ait. ${ }^{29}$

12. LIMOSELLA L. Mudwort

1. aquatica L. var. tenuifolia (Wolf) Pers.9.

14. ILYSANTHES Raf. False Pimpernel

1. dubia (L.) Barnhart. I. attenuata Small. ${ }^{34}$ I. riparia Raf. $^{29}$

2. anagallidea (Michx.) Robinson. ${ }^{29}$ I. gratioloides Benth. ${ }^{34}$ 
15. GRATIOLA L. Hedge Hyssop

1. virginiana L. ${ }^{29}$

4. aurea Muhl.29

17. VERONIGA (Tourn.) L. Speedwell

1. virginica L. ${ }^{1}$ (Culver's-root, Culver's Physic.)

4. Anagallis-aquatica L. (Water S.) V. Anagallis L. ${ }^{1}$

5. americana Schwein. ${ }^{29}$ (American Brooklime.)

7. scutellata L. ${ }^{29}$ (Marsh S.)

8. officinalis L. ${ }^{29}$ (Common S.)

*9. Chamaedrys L. ${ }^{29}$ (Bird's-eye.)

12. serpyllifolia L.29 (Thyme-leaved S.)

14. peregrina L. ${ }^{29}$ (Neckweed, Purslane S.)

*15. arvensis L. ${ }^{29}$ (Corn S.)

*16. agrestis L. ${ }^{*}$ (Field S.)

*18. hederaefolia L. ${ }^{1}$ (Ivy-leaved S.)

20. GERARDIA (Plumier) L. Gerardia

1. pedicularia L. ${ }^{29}$

3. flava L. ${ }^{29}$ (Downy False Foxglove.)

4. virginica (L.) B S P. (Smooth False Foxglove.) G. quercifolia Pursh. ${ }^{29}$

11. purpurea L. ${ }^{29-34}$ (Purple G.)

12. paupercula (Gray) Britton..$^{34}$ G. purpurea var. Gray..$^{29}$

13. maritima Raf. ${ }^{29}$ (Sea-side G.)

14. tenuifolia Vahl. ${ }^{29}$ (Slender G.)

17. parvifolia Chapm. ${ }^{32}$

22. CASTILleJA Mutis. Painted Cup

1. coccinea (L.) Spreng. ${ }^{29}$

24. MELAMPYRUM (Tourn.) L. Cow Wheat

1. lineare Lam. M. americanum Michx. ${ }^{25}$

27. PEDICULARIS (Tourn.) L. Lousewort

2. canadensis L. ${ }^{29}$ (Common L., Wood Betony.)

3. lanceolata Michx..$^{29}$

28. RHINANTHUS L. Yellow Rattle

2. Crista-galli L. ${ }^{29}$

FAMILY 143. LENTIBULARIACEAE (Bladderwort Family)

1. UTRICULARIA L. Bladderwort 
1. inflata Walt. ${ }^{28-34}$

2. clandestina Nutt. ${ }^{28-34}$

3. vulgaris L. ${ }^{28}$ (Greater B.) vulgaris var. americana Gray. U. Macrorhiza Le Conte.'

4. minor L. ${ }^{28}$ (Smaller B.)

5. gibba L. ${ }^{28-34}$

6. biflora Lam. ${ }^{28-34}$

8. intermedia Hayne. ${ }^{28-34}$

9. purpurea Walt. ${ }^{28-34}$

10. resupinata B. D. Greene..$^{11-28-34}$

12. subulata L. ${ }^{28-34}$

13. cornuta Michx. ${ }^{28-34}$

FAMILY 144. OROBANGHAGEAE (Broom-rape Family) 1. EPIFAGUS Nutt. Beech-drops. Cancer-root 1. virginiana (L.) Bart. ${ }^{28}$

2. CONOPHOLIS Wallr. Squaw-root. Cancer-root 1. americana (L. f.) Wallr. ${ }^{28}$

3. OROBANGHE (Tourn.) L. Broom-rape

5. uniflora L. ${ }^{28}$ (One-flowered Cancer-root.) Aphyllon uniflorum T. \& G. ${ }^{1}$

FAMILY 148. PHRYMACEAE (Lopseed Family)

1. PHRYMA L. Lopseed

1. leptostachya L. ${ }^{29}$

ORDER XLII PLANTAGINALES

FAMILY 149. PLANTAGINAGEAE (Plantain Family)

2. PLANTAGO (Tourn.) L. Plantain. Ribwort

2. major L. ${ }^{1}$ (Common P.)

3. Rugelii Dene. ${ }^{1}$

6. decipiens Barneoud. ${ }^{\perp}$ (Seaside P.)

*7. lanceolata L. ${ }^{1}$ (Rib Grass, Ripple Grass, English P.)

*8. media L. ${ }^{2}$ (Hoary P.)

10. aristata Michx. ${ }^{34}$ P. patagonica var. Gray. ${ }^{1-52}$

11. virginica L. $^{9}$

13. elongata Pursh. P. pusilla Nutt. ${ }^{1}$ 


\section{ORDER XLIII RUBIALES}

FAMILY 150. RUBIACEAE (Madder Family)

3. GALIUM L. Bedstraw. Cleavers

2. Aparine L. ${ }^{1}$ (Cleavers, Goose Grass.)

*3. tricorne Stokes. ${ }^{2}$

*5. verum L. ${ }^{1}$ (Yellow B.)

7. pilosum Ait. ${ }^{1}$

9. circaezans Michx. ${ }^{1}$ (Wild Liquorice.)

10. lanceolatum Torr. ${ }^{1}$ (Wild Liquorice.)

*14. Mollugo L. ${ }^{2}$

18. trifidum L. ${ }^{1-52}$ G. trifidum var. pusillum Gray. ${ }^{3}$

19. Claytoni Michx. ${ }^{41}$

20. tinctorium L. G. trifidum var. latifolium Torr. ${ }^{1}$

23. asprellum Michx..$^{34}$ (Rough B.)

24. triflorum Michx. ${ }^{1}$ (Sweet-scented B.)

6. MITCHELLA L. Patridge Berry

1. repens L. ${ }^{1}$

7. GEPHALANTHUS L. Buttonbush

1. occidentalis $\mathrm{L}^{9-34}$

8. HOUSTONIA L.

1. caerulea L. ${ }^{1}$ (Bluets, Innocence.)

6. tenuifolia Nutt. ${ }^{1}$

7. longifolia Gaertn. H. purpurea var. Gray. ${ }^{1}$

FAMILY 151. CAPRIFOLIACEAE (Honeysuckle Family)

1. DIERVILLA (Tourn.) Mill. Bush Honeysuckle

1. Lonicera Mill. ${ }^{24}$ D. trifida Moench. ${ }^{1}$

2. LONICERA L. Honeysuckle

1. caerulea L. var. villosa (Michx.) T. \& G. (Mountain Fly H.) L. caerulea L. ${ }^{24}$

*5. Xylosteum L. ${ }^{24}$ (European Fly H.)

6. oblongifolia (Goldie) Hook. ${ }^{1}$ (Swamp Fly H.)

9. sempervirens L. ${ }^{24}$ (Trumpet H.)

11. hirsuta Eat. (Hairy H.)

14. dioica L. ${ }^{24}$ L. glauca Hill. ${ }^{1}$

3. SYMPHORICARPOS (Dill.) Ludwig. Snowberry

1. orbiculatus Moench. ${ }^{24}$ (Indian Currant, Coral-berry.)

3. racemosus Michx. ${ }^{2-24}$ (Snowberry.) 
4. LINNAEA (Gronov.) L. Twin-flower

1. borealis L. var. americana (Forbes) Rehder. L. borealis Gronov. ${ }^{1}$

5. TRIOSTEUM L. Feverwort. Horse Gentian

1. perfoliatum L. ${ }^{1}$ (Tinker's Weed, Wild Coffee.)

2. aurantiacum Bicknell. ${ }^{24}$

6. VIBURNUM (Tourn.) L. Arrow-wood. Laurestinus

2. alnifolium Marsh. ${ }^{24}$ (Hobble-bush, Moosewood.) $V$. lantanoides Michx. ${ }^{1}$

5. acerifolium L. ${ }^{24}$ (Dockmackie, Arrow-wood.)

7. molle Michx. ${ }^{52}$

10. dentatum L. ${ }^{24-34}$ (Arrow-wood.)

11. cassinoides L. ${ }^{24-34}$ (Withe-rod, Wild Raisin.)

12. nudum $L .{ }^{1}$

13. Lentago L. ${ }^{2}$ (Sweet Viburnum, Sheepberry, Nannyberry)

7. SAMBUCUS (Tourn.) L. Elder

1. canadensis L..$^{24}$ (Common E.)

2. racemosa L. ${ }^{24}$ (Red-berried E.)

FAMILY 153. DIPSACACEAE (Teasel Family)

1. DIPSACUS (Tourn.) L. Teasel

*1. sylvestris Huds. (Wild T.)

ORDER XLIV CAMPANULALES

FAMILY 154. CUCURBITACEAE (Gourd Family)

2. SICYOS L. One-seeded Bur Cucumber

1. angulatus $L .{ }^{1}$

3. ECHINOCYSTIS T. \& G. Wild Balsam-apple

1. lobata (Michx.) T. \& G. ${ }^{1}$

FAMILY 155. CAMPANULACEAE (Bluebell Family)

1. SPECULARIA (Heist.) Fabricius. Venus's Lookingglass

1. perfoliata (L.) A. D C. ${ }^{15}$

2. CAMPANULA (Tourn.) L. Bellflower

*3. rapunculoides $L .{ }^{15}$

7. aparinoides Pursh..$^{15-34}$ (Marsh B.) 


\section{JASIONE L. Sheep's-bit}

*1. montana L. ${ }^{15-17}$

FAMILY 156. LOBELIACEAE (Lobelia Family)

1. LOBELIA (Plumier) L.

1. cardinalis L. ${ }^{15-34}$ (Cardinal Flower.)

7. spicata Lam. ${ }^{15}$

11. inflata L. ${ }^{15-34}$ (Indian Tobacco.)

13. Dortmanna L. ${ }^{15-34}$ (Water Lobelia.)

FAMILY 157. COMPOSITAE (Composite Family)

1. VERNONIA Schreb. Ironweed

2. noveboracensis Willd. ${ }^{34}$

\section{SCLEROLEPIS Cass.}

1. uniflora (Walt.) B S P. ${ }^{40}$

4. EUPATORIUM (Tourn.) L. Thoroughwort

1. purpureum L. ${ }^{1}$ (Joe-Pye Weed, Trumpet Weed.) purpureum var. maculatum (L.) Darl.1

6. hyssopifolium L. ${ }^{11}$

10. verbenaefolium Michx. E. teucrifolium Willd. ${ }^{52}$

11. rotundifolium L. ${ }^{1}$

12. pubescens Muhl. E. rotundifolium var. ovatum Torr. ${ }^{1}$

13. sessilifolium L. ${ }^{1}$ (Upland Boneset.)

14. perfoliatum L. ${ }^{1}$ (Thoroughwort, Boneset.)

16. urticaefolium Reichard. (White Snakeroot. (E. ageratoides L. . ${ }^{1}$

17. aromaticum L. ${ }^{1}$

5. MIKANIA Willd. Climbing Hemp-weed

1. scandens (L.) Willd. ${ }^{1}$

9. LIATRIS Schreb. Button Snakeroot. Blazing Star 5. scariosa Willd. ${ }^{1}$

10. GRINDELIA Willd. Gum-plant. Tar-weed

1. squarrosa (Pursh.) Dunal. ${ }^{2}$

14. CHRYSOPSIS Nutt. (Golden Aster.)

2. falcata (Pursh.) Ell. ${ }^{34}$

15. SOLIDAGO L. Golden-rod

3. caesia L. ${ }^{1}$ 
3. caesia var. axillaris (Pursh.) Gray.

4. latifolia L. ${ }^{1}$

6. bicolor L. ${ }^{1}$

7. hispida Muhl. S. bicolor var. concolor T. \& G. ${ }^{1}$

15. racemosa Greene. racemosa var. Gillmani (Gray) Fernald.

16. decumbens Greene.

17. puberula Nutt. ${ }^{3 \pm}$

18. stricta Ait.

19. uliginosa Nutt. ${ }^{34}$

20. speciosa Nutt. ${ }^{\text {I }}$ speciosa var. angustata T. \& G.

21. sempervirens L. ${ }^{5-55}$

23. arguta Ait. ${ }^{1}$

21. Boottii Hook.

25. juncea Ait. ${ }^{1}$

26. neglecta $T$. \& G. ${ }^{1}$

27. uniligulata (D C.) Porter. S. neglecia var. linoides Gray. ${ }^{1}$ 28. odora Ait. ${ }^{34}$ (Sweet Golden-rod.)

29. tortifolia Ell.

31. Elliottii T. \& G. ${ }^{1}$

32. ulmifolia Muhl. ${ }^{1}$

33. rugosa Mill. ${ }^{1}$

38. nemoralis Ait. ${ }^{1-52}$

11. canadensis L. $^{1}$

44. serotina Ait. ${ }^{1}$ serotina var. gigantea (Ait.) Gray.1

46. rigida L. ${ }^{1}$

50. graminifolia (L.) Salisb. S. lanceolatc L. ${ }^{1}$

53. tenuifolia Pursh. ${ }^{10}$

22. ASTER (Tourn.) L. Starwort. Frost-flower. Aster 2. divaricatus L. A. corymbosus tit. ${ }^{1}$

6. macrophyllus L. ${ }^{1}$

7. Herveyi Gray. ${ }^{1}$

8. spectabilis Ait. ${ }^{1}$

11. radula Ait. ${ }^{1}$

14. norae-angliae L. ${ }^{1}$ norae-angliae var. roseus (Desf.) D C. 
17. concolor L. ${ }^{1}$

18. patens Ait. ${ }^{1}$ patens var. phlogifolius Nees.

22. undulatus L. ${ }^{1}$

23. cordifolius L. ${ }^{1}$

25. sagittifolius Wedemeyer. ${ }^{I}$

29. laevis L. ${ }^{1-52}$

33. ericoides L. ${ }^{1}$

35. amethystinus Nutt. ${ }^{1}$

36. multiflorus Ait. ${ }^{1}$

38. dumosus L. ${ }^{1}$

39. vimineus $\mathrm{Lam} \cdot{ }^{1-52}$ vimineus var. (foliolosus) (Ait.) Gray. ${ }^{\mathrm{I}}$

40. lateriflorus (L.) Britton. A. diffusus Ait. ${ }^{1}$

41. tradescanti L. ${ }^{1}$

42. paniculatus Lam. ${ }^{1}$

43. salicifolius Ait. ${ }^{1}$

44. junceus Ait. ${ }^{1}$

45. longifolius Lam. ${ }^{1}$

46. novi-belgii L. ${ }^{1}$ novi-belgii var. elodes (T. \& G.) Gray. ${ }^{1}$

49. puniceus L. ${ }^{1}$

puniceus var. firmus (Nees.) T. \& G. var. laevicaulis Gray.' puniceus var. lucidulus Gray. ${ }^{1}$

50. umbellatus Mill. ${ }^{I}$

51. infirmus Michx. ${ }^{1}$

52. linariifolius L. ${ }^{1}$

54. acuminatus Michx."

55. nemoralis Ait. ${ }^{1}$ nemoralis var. Blakei Porter.

56. tenuifolius L. ${ }^{1}$

57. subulatus Michx. ${ }^{1}$

23. ERIGERON L. Fleabane

3. pulchellus Michx. (Robin's Plantain.) E. bellidifolius Michx. ${ }^{1}$

4. philadelphicus L. ${ }^{1}$

6. annuus (L.) Pers. ${ }^{1}$ (Daisy F., Sweet Scabious.)

7. ramosus (Walt.) B S P. ${ }^{52}$ (Daisy F.) E. strigosus Muhl.1 ramosus var. discoideus (Robbins) B S P. I 
8. canadensis L. ${ }^{1-52}$ (Horse-weed, Butter-weed.)

24. SERIOCARPUS Nees. White-topped Aster

1. asteroides (L.) B S P. S. conyzoides Nees. ${ }^{1}$

2. linifolius (L.) B S P. S. solidagineus Nees. ${ }^{1}$

26. PLUCHEA Cass. Marsh Fleabane

2. camphorata (L.) D C. ${ }^{1-52}$ (Salt Marsh Fleabane.)

28. ANTENNARIA Gaertn. Everlasting. Ladies' Tobacco. Pussy's Toes

A. Parlinii var. ambigua Fernald. ${ }^{3-6}$

3. plantaginifolia (L.) Richards. (Plantain-leaved E.) A. plantaginea $\mathrm{R}$. $\mathrm{Br}^{3-6}$

plantaginifolia var. petiolata Fernald..$^{3-6}$

4. fallax Greene. A. ambigens Fernald. ${ }^{6}$

8. neodioica Greene. ${ }^{3-6}$

10. neglecta Greene. ${ }^{3-6}$

29. ANAPHALIS D C. Everlasting

1. margaritacea (L.) B. \& H. ${ }^{34}$ (Pearly E.)

30. GNAPHALIUM L. Cudweed

1. polycephalum Michx. ${ }^{1}$ (Common Everlasting.)

2. decurrens Ives. ${ }^{1}$ (Everlasting.)

3. uliginosum L. ${ }^{1}$ (Low C.)

6. purpureum L. ${ }^{1}$ (Purplish C.)

31. INULA L. Elecampane

*1. Helenium L. ${ }^{1}$ (Elecampane.)

39. IVA L. Marsh Elder. Highwater-shrub

1. oraria Bartlett. I. frutescens L. ${ }^{1}$

40. AMBROSIA (Tourn.) L. Ragweed

3. artemisiifolia L. ${ }^{1}$ (Roman Wormwood, Hog-weed, Bitter-weed.)

42. XANTHIUM (Tourn.) L. Cocklebur. Clotbur

*1. Spinosum L. ${ }^{1}$

2. canadense Mill. ${ }^{52}$

6. echinatum Murr. X. canadense var. Gray. ${ }^{1}$

46. RUDBECKIA L. Cone Flower

3. hirta L.' (Yellow Daisy, Black-eyed Susan, Niggerhead.)

7. laciniata L. ${ }^{1}$ 
51. HELIANTHUS L. Sunflower

1. annuus L. ${ }^{2}$ (Common S.)

2. petiolaris Nutt. ${ }^{2}$

10. mollis Lam. $^{32}$

14. giganteus $\mathrm{L}$.

19. divaricatus L. ${ }^{1}$

21. strumosus L. ${ }^{1}$

23. decapetalus L. ${ }^{1}$

24. tuberosus L. ${ }^{1}$ (Jerusalem Artichoke.)

53. VERBESINA L. Crownbeard

*4. encelioides (Cav.) B. \& H. var. exauriculata Robinson \& Greenman. B. U.

54. COREOPSIS L. Tickseed

1. rosea Nutt. ${ }^{11}$

C. cardaminefolia T. \& G. ${ }^{2}$

56. BIDENS L. Bur Marigold

3. discoidea (T. \& G.) Britton. ${ }^{32}$ Coreopsis T. \& G. ${ }^{1}$

4. frondosa L. ${ }^{32}$ (Beggar-ticks.)

5. vulgata Greene..$^{32}$ (Beggar-ticks, Stick-tight.)

6. comosa (Gray) Wiegand. ${ }^{32}$

7. connata Muhl. ${ }^{1}$ (Swamp Beggar-ticks.)

8. cernua L. ${ }^{1}$ (Stick-tight.)

9. laevis (L.) B S P. ${ }^{34}$ B. chrysanthemoides Michx. ${ }^{1}$

10. bipinnata L. ${ }^{1}$ (Spanish Needles.)

12. trichosperma (Michx.) Britton. Coreopsis Michx. ${ }^{1}$

15. Beckii Torr. ${ }^{1}$ (Water Marigold.)

59. GALINSOGA R. \& P.

*1. parviflora Cav. ${ }^{1}$

* . parviflora var. hispida D C. ${ }^{2}$

64. HELENIUM L. Sneezeweed

1. nudiflorum Nutt. ${ }^{14}$

2. autumnale L. ${ }^{1}$

3. tenuifolium Nutt. B. U.

67. ACHILLEA (Vaill.) L. Yarrow

3. Millefolium L. ${ }^{1}$ (Common Y., Milfoil.)

68. ANTHEMIS (Mich.) L. Chamomile

*1. Cotula L. ${ }^{1-52}$ (May-weed, Dog Fennel.) 
*2. arvensis L. ${ }^{1} \quad$ (Corn C.)

*3. nobilis L. ${ }^{1}$ (Garden C.)

*4. tinctoria L. (Yellow C.)

70. CHRYSANTHEMUM (Tourn.) L. Ox-eye Daisy *1. Leucanthemum L. ${ }^{1}$ (Ox-eye or White Daisy, Marguerite, White-weed.)

*3. Parthenium (L.) Bernh. ${ }^{1}$ (Feverfew.)

*4. Balsamita L. var. tanacetoides Boiss. ${ }^{1}$ (Costmary, Mint Geranium.)

71. TANACETUM L. Tansy

*1. vulgare L. ${ }^{1}$ (Common T.) vulgare var. crispum D C..$^{30}$

73. ARTEMISIA L. Wormwood

1. caudata Michx. ${ }^{1}$

8. ludoviciana Nutt. ${ }^{1}$ (Western Mugwort, White Sage.)

*9. vulgaris L. ${ }^{1}$ (Common Mugwort.)

*10. kansana Britton. ${ }^{2}$

*11. Stelleriana Bess. ${ }^{2-3}$ (Beach W., Dusty Miller, Old Woman.)

12. biennis Willd. ${ }^{I}$

*14. Absinthium L. ${ }^{1}$ (Wormwood.)

74. TUSSILAGO (Tourn.) L. Coltsfoot

*1. Farfara L. ${ }^{1}$

77. ERECHTITES Raf. Fireweed

1. hieracifolia (L.) Raf. ${ }^{1-52}$

78. CACALIA L. Indian Plantain

1. suaveolens $\mathrm{L}^{\mathrm{I}}$

79. SENECIO (Tourn.) L. Groundsel. Ragwort. Squaw-weed

*1. vulgaris L. ${ }^{1}$ (Common Groundsel.)

*2. viscosus L. ${ }^{1}$

8. obovatus Muhl. S. aureus var. T. $\& G^{1}$

9. aureus L.' (Golden Ragwort.)

11. Balsamitae Muhl. S. aureus var. T. \& $G .{ }^{1}$

80. ARCTIUM L. Burdock

*1. Lappa L. (Great B.) A. Lappa var. Majus Gray. ${ }^{1}$

*2. minus Bernh. (Common B.) A. Lappa var. Gray. ${ }^{1}$ 
82. CARDUUS (Tourn.) L. Plumeless Thistle

*1. nutans L. ${ }^{2}$ (Musk Thistle.)

$* 2$. acanthoides L. ${ }^{41}$

83. CIRSIUM (Tourn.) Hill. Common or Plumed Thistle

*1. lanceolatum (L.) Hill. (Common or Bull T.) Cnicus lanceolatus Hoffm. ${ }^{1-52}$

2. spinosissimum (Walt.) Scop. (Yellow T.) Cnicus horridulus Pursh..$^{1-52}$

6. discolor (Muhl.) Spreng. Cnicus altissimus var. Gray. ${ }^{1-52}$

7. altissimum (L.) Spreng. Cnicus altissimus Willd. ${ }^{1}$

10. muticum Michx. (Swamp Thistle.) Cnicus muticus Pursh. ${ }^{1}$

11. pumilum (Nutt.) Spreng. (Pasture or Bull Thistle.) Cnicus pumilus Torr. ${ }^{1}$

*13. arvense (L.) Scop. (Canada Thistle.) Cnicus arvensis Hoffm. ${ }^{1-52}$

84. ONOPORDUM (Vaill.) L. Cotton or Scotch Thistle *1. Acanthium L. ${ }^{1}$

86. GENTAUREA L. Star Thistle

*4. Jacea L. ${ }^{1}$

*5. Cyanus L. ${ }^{1}$ (Bluebottle, Bachelor's Button, Corn-flower)

*7. nigra L. ${ }^{1}$ (Knapweed, Spanish Buttons.)

8. americana Nutt.

88. LAPSANA L. Nipple-wort

*1. communis L. Lampsana Tourn. ${ }^{1}$

91. CICHORIUM (Tourn.) L. Succory or Chicory

*1. Intybus L. ${ }^{1-52}$ (Common C., Blue Sailors.)

92. KRIGIA Schreb. Dwarf Dandelion

2. virginica (L.) Willd. ${ }^{1}$

93. HYPOCHAERIS L. Cat's-ear

*1. radicata L. .1

94. LEONTODON L. Hawkbit

*1. autumnalis L. ${ }^{34}$ (Fall Dandelion, "Arnica.") 
98. TARAXACUM (Haller) Ludwig. Dandelion

*1. officinale Weber. ${ }^{30}$ (Common D.)

*2. erythrospermum Andrz. ${ }^{21-30}$ (Red-seeded D.)

99. SONGHUS (Tourn.) L. Sow Thistle

${ }^{*} 1$. arvensis L. ${ }^{1}$ (Field S.)

*2. oleraceus L. ${ }^{1}$ (Common S.)

*3. asper (L.) Hill. ${ }^{1}$ (Spiny-leaved S.)

109. LACTUCA (Tourn.) L. Lettuce

*1. scariola L. ${ }^{2}$ (Prickly L.)

3. canadensis L. ${ }^{1}$ (Wild L., Horse-weed.)

4. integrifolia Bigel. ${ }^{1}$

6. hirsuta Muhl. ${ }^{1}$

14. spicata (Lam.) Hitchc. L. leucophoea Gray. ${ }^{1}$

105. PRENANTHES (Vaill.) L. Rattlesnake-root

6. alba L. ${ }^{1}$ (White Lettuce, Rattlesnake-root.)

7. serpentaria Fursh. ${ }^{1}$ (Lion's-foot, Gall-of-the-earth.)

10. altissima L. ${ }^{1}$

106. HIERACIUM (Tourn.) L. Hawkweed

*2. aurantiacum L. ${ }^{1}$ (Grange H., Devil's Paint-brush, Grim the Collier.)

7. venosum L. ${ }^{1}$ (Rattlesnake-weed, Poor Robin's Plantain)

9. paniculatum $L .{ }^{1}$

10. marianum Willd. ${ }^{1}$

11. scabrum Michx. ${ }^{34}$

12. Gronovii L. ${ }^{34}$

16. canadense Michx. ${ }^{3}$ 
References. Literature cited as authority.

1. Plants of Rhode Island.

James Lawrence Bennett. 1888.

2. Rhode Island Plant Notes. 1. Rhodora 1- 46 J. Franklin Collins.

3. Rhode Island Plant Notes. $2 . \quad$ " 1-105 J. Franklin Collins.

4. Preliminary Lists of New England Plants. “ 1-93 1. Walter Deane.

5. Preliminary Lists of New England Plants. " 1-159 2. Walter Deane.

6. Preliminary Lists of New England Plants. " 1-160 3. M. L. Fernald.

7. Preliminary Lists of New England Plants. 4. B. L. Robinson.

8. Preliminary Lists of New England Plants. 6. J. R. Churchill.

9. The Old Time Flora of Providence.

W. Whitman Bailey.

10. Solidago Tenuifolia, a Weed in Rhode Island. W. Whitman Bailey.

11. Notes on the Flora of Rhode Island.

W. Whitman Bailey.

12. The New England Species of the Genus Panicum. F. Lamson Scribner \& Elmer D. Merrill.

13. Notes on Ericaceae of New England. Walter Deane.

14. Preliminary Lists of New England Plants. 7. E. B. Chamberlain.

15. Preliminary Lists of New England Plants. “ 4- 15 8. Emile F. Williams.

16. Some Notable Plant Stations in Rhode Island. W. Whitman Bailey.

17. Jasione montana in Massachusetts. Anna M. Scorgie.

18. Preliminary Lists of New England Plants. “ 4-203 9. Wm. P. Rich.

19. Preliminary Lists of New England Plants. " 4-218 10. M. L. Fernald.

20. Preliminary Lists of New England Plants. “ 5-188 12. B. L. Robinson. 
21. Corallorrhiza innata \& Jaraxacum ery- Rhodora 5-291 throspermum in Rhode Island. J. Franklin Collins.

22. Preliminary Lists of New England Plants. 13. M. L. Fernald.

23. Black Spruce in Rhode Island. J. Franklin Collins.

24. Preliminary Lists of New England Plants. 14. Alfred Rehder.

25. Saururus cernuus. S. N. F. Sanford.

26. Preliminary Lists of New England Plants. 15. Arthur Stanley Pease.

27. Some Interesting Rhode Island Bogs. J. Franklin Collins.

28. Preliminary Lists of New England Plants. 16. Walter Deane.

29. Preliminary Lists of New England Plants. 17. J. R. Churchill.

30. Remarks on Rhode Island Plants. Frederick S. Beattie.

31. Lotus tenuis \& Lycium vulgare Dunal. Reginald Heber Howe, Jr.

32. 12 Additions to the Flora of Rhode Island. M. L. Fernald.

33. Preliminary Lists of New England Plants. 20. M. L. Fernald \& A. J. Eames.

34. Flora of the Great Swamp in Rhode Island. Ernest Shaw Reynolds.

35. Scirpus hudsonianus in Rhode Island. Ernest Shaw Reynolds.

36. Addenda to Preliminary Lists. 15. Arthur Stanley Pease.

37. Plants of Tiverton, Rhode Island. Myra M. Sampson.

38. Preliminary Lists of New England Plants. 21. M. L. Fernald.

39. Preliminary Lists of New England Plants. 22. M. L. Fernald.

40. Sclerolepis uniflora near Wallum Pond. J. Franklin Collins.

41. Additions to Rhode Island Flora. $6-34$ 6- 41 $6-54$ $6-77$ $6-85$ $6-149$ $6-151$ $7-33$ $7-39$ $7-167$ $8-219$ $9-86$ $9-117$ $10-20$ $10-36$ $10-112$

$10-135$ $10-168$ $12-13$ $12-216$ M. L. Fernald.

42. Cyperus Grayii in Rhode Island. R. W. Woodward.

43. Arenaria caroliniana and A. squarrosa at Weekapaug, R. I. John H. Sage. 
44. Juncus dichotomus in Rhode Island. Rhodora 15-151 R. W. Woodward.

45. Preliminary Lists of New England Plants. “ 18-245 24. Clarence H. Knowlton.

46. Preliminary Lists of New England Plants. “ 19-217 25. Clarence H. Knowlton.

47. Preliminary Lists of New England Plants. " 20-182 26. Clarence H. Knowlton.

48. Some Rhode Island Grasses. R. W. Woodward.

49. Some Species \& Varieties of Elymus in " $20-81$ Eastern North America. K. M. Wiegand.

50. The Genus Puccinellia in Eastern North " $18-1$ America. Fernald \& Wetherby.

51. Notes on the Rhode Island Flora. J. Franklin Collins, Bulletin of Torrey Botanical Club. 20.-240.

52. Notes on the Flora of Block Island. IV. W. Bailey. Bulletin of Torrey Botanical Club. 20-227.

53. The Genus Sabatia in New England. Rhodora 18-145 M. L. Fernald. 



B-F Librars

JUN 101980 


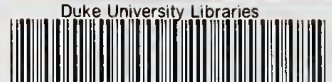 (ling D00262395R}

